Pacific Journal of Mathematics

UNITARY COBORDISM OF CLASSIFYING SPACES OF 


\title{
UNITARY COBORDISM OF CLASSIFYING SPACES OF QUATERNION GROUPS
}

\author{
Abdeslam Mesnaoui
}

The main purpose of this article is to prove that the complex cobordism ring of classifying spaces of quaternion groups $\Gamma_{k}\left(\left|\Gamma_{k}\right|=2^{k}\right)$ is a quotient of the graded ring $U^{*}(p t)[[X, Y, Z]](\operatorname{dim} X=\operatorname{dim} Y=$ $2, \operatorname{dim}=Z=4$ ) by a graded ideal generated by six homogeneous formal power series.

0. Introduction. Let $\Gamma_{k}$ be the generalized quaternion group. $\Gamma_{k}$ is generated by $u, v$, subject to the relations $u^{t}=v^{2}, u v u=v, t=$ $2^{k-2}$. In order to calculate $U^{*}\left(B \Gamma_{k}\right)$ we first consider the case $k=3$, i.e. $\Gamma_{3}=\Gamma$. We recall that $\Gamma=\{ \pm 1, \pm i, \pm j, \pm k\}$ with the relations $i^{2}=j^{2}=k^{2}=-1, i j=k, j k=i, k i=j$. We shall define $A \in \tilde{U}^{2}(B \Gamma), B \in \tilde{U}^{2}(B \Gamma), D \in \tilde{U}^{4}(B \Gamma)$ as Euler classes of complex vector bundles over $B \Gamma$ corresponding to unitary irreducible representations of $\Gamma$. Let $\Lambda_{*}$ be the graded $U^{*}(p t)$-algebra $U^{*}(p t)[[X, Y, Z]]$ with $\operatorname{dim} X=\operatorname{dim} Y=2, \operatorname{dim} Z=4, \Omega_{*}=U^{*}(p t)[[Z]] \subset \Lambda_{*}$ and $U^{*}(p t)[[D]]=\left\{P(D), P \in \Omega_{*}\right\}$. Then by using the Atiyah-Hirzebruch spectral sequence we obtain the following results where $T(Z) \in \Omega_{4}$, $J(Z) \in \Omega_{0}$ are well defined formal power series.

THEOREM 2.18. (a) As graded $U^{*}(p t)$-algebras we have:

$$
U^{*}(p t)[[D]] \simeq \Omega_{*} /(T(Z)) .
$$

(b) As graded $U^{*}(p t)[[D]]-$ modules we have: $U^{*}(B \Gamma) \simeq U^{*}(p t)[[D]]$ $\oplus U^{*}(p t)[[D]] \cdot A \oplus U^{*}(p t)[[D]] \cdot B$ and $A, B$ have the same annihilator $(2+J(D)) \cdot U^{*}(p t)[[D]]$.

THEOREM 2.17. The graded $U^{*}(p t)$-algebra $U^{*}(B \Gamma)$ is isomorphic to $\Lambda_{*} / I_{*}$ where $I_{*}$ is a graded ideal generated by six homogeneous formal power series.

The method used for $\Gamma$ is extended to $\Gamma_{k}, k \geq 4$. As before we shall define $B_{k} \in \tilde{U}^{2}\left(B \Gamma_{K}\right), C_{k} \in \tilde{U}^{2}\left(B \Gamma_{k}\right), D_{k} \in \tilde{U}^{4}\left(B \Gamma_{k}\right)$ as Euler classes of complex vector bundles over $B \Gamma_{k}$ corresponding to unitary irreducible representations of $\Gamma_{k}$ and elements $G^{\prime}(Z) \in \Omega_{2}$, 
$T_{k}(Z) \in \Omega_{4}$. If $B_{k}^{\prime}=B_{k}+G_{k}^{\prime}\left(D_{k}\right), C_{k}^{\prime}=C_{k}+G_{k}^{\prime}\left(D_{k}\right)$ then we get: bras.

TheOREM 3.14. (a) $U^{*}(p t)\left[\left[D_{k}\right]\right] \simeq \Omega_{*} /\left(T_{k}\right)$ as graded $U^{*}(p t)$-alge-

(b) As graded $U^{*}(p t)\left[\left[D_{k}\right]\right]-$ modules we have:

$$
U^{*}\left(B \Gamma_{k}\right) \simeq U^{*}(p t)\left[\left[D_{k}\right]\right] \oplus U^{*}(p t)\left[\left[D_{k}\right]\right] \cdot B_{k}^{\prime} \oplus U^{*}(p t)\left[\left[D_{k}\right]\right] \cdot C_{k}^{\prime}
$$

and $B_{k}^{\prime}, C_{k}^{\prime}$ have the same annihilator $\left(2+J\left(D_{k}\right)\right) \cdot U^{*}(p t)\left[\left[D_{k}\right]\right]$.

THEOREM 3.12. The graded $U^{*}(p t)$-algebra $U^{*}\left(B \Gamma_{k}\right)$ is isomorphic to $\Lambda_{*} / \tilde{I}_{*}$ where $\tilde{I}_{*}$ is a graded ideal of $\Lambda_{*}$ generated by six homogeneous formal power series.

In the appendix, part $A$, we give a new method of calculating $U^{*}\left(B \mathbb{Z}_{m}\right)$. Let $\Lambda_{*}^{\prime}$ be the graded algebra $U^{*}(p t)[[Z]], \operatorname{dim} Z=2$.

TheOREM A.1. $U^{*}\left(B \mathbb{Z}_{m}\right) \simeq \Lambda_{*}^{\prime} /([m](Z))$ as graded $U^{*}(p t)$-algebras.

In part $B$ we show that:

TheOREM B.2.

$$
U^{2 i+2}(B S U(n)) \simeq U^{2 i+2}(B U(n)) / e\left(\Lambda^{n} \gamma(n)\right) \cdot U^{2 i}(B U(n))
$$

and $U^{2 i+1}(B S U(n))=0, i \in \mathbb{Z}$.

In this theorem $e\left(\Lambda^{n} \gamma(n)\right)$ is the Euler class of $\Lambda^{n} \gamma(n)$ where $\gamma(n)$ denotes the universal bundle over $B U(n)$.

In part $\mathrm{C}$ we calculate $H^{*}\left(B \Gamma_{k}\right), k \geq 4$.

Theorem C. If $k \geq 4$ then we have $H^{*}\left(B \Gamma_{k}\right)=\mathbb{Z}\left[x_{k}, y_{k}, z_{k}\right]$ with $\operatorname{dim} x_{k}=\operatorname{dim} y_{k}=2, \operatorname{dim} z_{k}=4$, subject to the relations:

$$
2 x_{k}=2 y_{k}=x_{k} y_{k}=2^{k} z_{k}=0, \quad x_{k}^{2}=y_{k}^{2}=2^{k-1} z_{k} \text {. }
$$

Theorem $\mathrm{C}$ is certainly known to workers in the field.

The layout is as follows:

I Preliminaries and notations.

II Calculation of $U^{*}(B \Gamma)$.

III Calculation of $U^{*}\left(B \Gamma_{k}\right), k \geq 4$.

IV Appendix. 
In the course of the computations we have determined the leading coefficients of some formal power series with the purpose of using them in a subsequent paper where the bordism groups $\tilde{U}_{*}\left(B \Gamma_{k}\right)$ are calculated.

We shall use the same notation for unitary irreducible representations of $\Gamma_{k}$ and corresponding complex vector bundles over $B \Gamma_{k}$. The notation $\gamma(n)$ will be used for the universal complex vector bundle over $B U(n)$. The notation $\mathbb{Z}$ will be for the ring of integers and $\mathbb{C}$ for the complex number field.

The results of this paper have been obtained in 1983 under the supervision of Dr. L. Hodgkin, University of London. I thank him sincerely for having proposed the subject, for his advice and encouragement. I would like to express my deep thanks to the referee who made many useful suggestions; they helped to improve the exposition of this paper and the statement of some results, particularly Theorems 2.18 and 3.14 .

I. Preliminaries and notations. 1 . Let $X$ be a CW-complex; we define a filtration on $U^{n}(X)$ by the subgroups

$$
J^{p, q}=\operatorname{Ker}\left(i^{*}: U^{n}(X) \rightarrow U^{n}\left(X_{p-1}\right)\right),
$$

$X_{p}$ being the $p$-skeleton of $X, i: X_{p-1} \subset X, p+q=n ; U^{n}(X)$ is a topological group, the subgroups $J^{p, q}$ being a fundamental system of neighbourhoods of 0 ; we denote this topology by $T$. If the $U^{*}$-AtiyahHirzebruch spectral sequence (denoted by $U^{*}$-AHSS) for $X$ collapses then $T$ is complete and Hausdorff (see [3]). The edge homomorphism $\mu: U^{n}(X)-H^{n}(X)$ is defined by $\mu=0$ if $n<0$ and if $n \geq 0$ it is the projection $U^{n}(X)=J^{0, n}=J^{n, 0} \rightarrow J^{n, 0} / J^{n+1,-1}=E_{\infty}^{n, 0} \subset E_{2}^{n, 0}=$ $H^{n}(X)$. By easy arguments involving spectral sequences we have the following basic result:

THEOREM 1.1. Let $X$ be a $C W$-complex such that:

(a) The $U^{*}$-AHSS for $X$ collapses.

(b) For each $n \geq 0$ there are elements $a_{\text {in }}$ generating the $\mathbb{Z}$-module $H^{n}(X)$.

Then for each $n \geq 0$ there are elements $A_{\text {in }} \in U^{n}(X)$ such that:

(a) $\mu\left(A_{\text {in }}\right)=a_{\text {in }}$.

(b) If $E$ denotes the $U^{*}(p t)$-submodule of $U^{*}(X)$ generated by the system $\left(A_{i n}\right)$ and if $E_{n}$ is the $n$-component of $E$ then $\bar{E}_{n}=U^{n}(X), \bar{E}_{n}$ being the closure of $E_{n}$ for $T$. 
Moreover (b) is valid of we take any system $\left(A_{\text {in }}^{\prime}\right), A_{\text {in }}^{\prime} \in U^{n}(X)$ such that $\mu\left(A_{i n}^{\prime}\right)=a_{\text {in }}$ for each $(i, n)$.

(See Theorem 2.5 for a proof of this result in a special case.)

2. Let $X$ be a skeleton-finite CW-complex, which is the case we are interested in. There is a ring spectra map $f: M U \rightarrow H$ (see [1]); by naturality of AHSS the map $f^{\#}(X): U^{*}(X) \rightarrow H^{*}(X)$ induced by $f$ is identical to the edge-homomorphism described above. Let $\xi$ be a complex vector bundle over $X$ of dimension $n$; the Conner-Floyd characteristic classes of $\xi$ will be denoted by $c f_{i}(\xi)$; the Euler class $e(\xi)$ of $\xi$ for $M U$ is $c f_{n}(\xi)$ and the Euler class $e_{1}(\xi)$ for $H$ is the Chern class $c_{n}(\xi)$. As $f^{\#}(X)$ maps Euler classes on Euler classes we have $\mu(e(\xi))=e_{1}(\xi)$ (see [7]).

3. Consider the formal power series ring $E_{*}=U^{*}(p t)\left[\left[c_{1}, c_{2}, \ldots, c_{r}\right]\right]$ graded by taking $\operatorname{dim} c_{1}=n_{1}>0, \ldots, \operatorname{dim} c_{r}=n_{r}>0$. Given $P\left(c_{1}, \ldots, c_{r}\right) \in E_{n}$ with $P \neq 0$,

$$
P=\sum a_{u} \cdot c_{1}^{u_{1}} \cdots c_{r}^{u_{r}}, \quad u=\left(u_{1}, \ldots, u_{r}\right),
$$

we define $\nu(P)=\left\{\inf \left(n_{1} u_{1}+\cdots+n_{r} u_{r}\right), a_{u} \neq 0\right\}$ and $\nu(0)=+\infty$. Let $J_{p}$ be $\left\{P \in E_{n} \mid \nu(P) \geq p\right\}$; we have $E_{n}=J_{0} \supset J_{1} \supset \cdots$, and since $\bigcap_{p \geq 0} J_{p}=0, E_{n}=\operatorname{Lim} E_{n} / J_{p}$, it follows that $E_{n}$ is complete and Hausdorff for the topology defined by the filtration $\left(J_{p}\right)$.

Suppose that $B$ is a $\mathrm{CW}$ complex such that the associated $U^{*}$ AHSS collapses; if $A_{i} \in U^{n_{i}}(B), i=1,2, \ldots, r$, then there is a unique continuous homomorphism $\psi: E_{*} \rightarrow U^{*}(B)$ such that $\psi\left(c_{i}\right)=A_{i}$, $i=1,2, \ldots, r$.

Now in a different situation consider the case where $B_{1}$ is a CWcomplex such that $U^{*}\left(B_{1}\right)=E_{*}$. There are two topologies on $U^{*}\left(B_{1}\right)$ defined respectively by the filtration $\left(J_{p}\right)$ on $E_{*}$ and by the filtration $\left(J_{1}^{p, q}\right)$ deduced from the $U^{*}$-AHSS for $B_{1}$. If $B$ is a CW-complex such that the $U^{*}$-AHSS for $B$ collapses, $\left(J^{p, q}\right)$ the corresponding filtration on $U^{*}(B)$ (see $\S \mathrm{I}$ ) and $g$ a continuous map: $B \rightarrow B_{1}$ then from $J_{p} \subset$ $J_{1}^{p, q}, g^{*}\left(J_{1}^{p, q}\right) \subset J^{p, q}$ it follows that $g^{*}: E_{n} \rightarrow U^{n}(B)$ is continuous for the topologies defined by $\nu$ on $E_{n}$ and $\left(J^{p, q}\right)$ on $U^{*}(B)$. As a consequence if $\left(P_{m}\right)$ is a sequence of polynomials such that $\left(P_{m}\right) \rightarrow P$ in $E_{n}$ and if $g^{*}\left(c_{i}\right)=A_{i}$ then $P_{m}\left(A_{1}, \ldots, A_{r}\right) \rightarrow g^{*}(P)$ in $U^{*}(B)$; so if $P=\sum a_{u} c_{1}^{u_{1}} \cdots c_{r}^{u_{r}} \in E_{n}$ we can write $g^{*}(P)=\sum a_{u} A_{1}^{u_{1}} \cdots A_{r}^{u_{r}}$.

In the sequel we shall also be concerned with $\Lambda_{*}=U^{*}(p t)[[X, Y, Z]]$, $\operatorname{dim} X=\operatorname{dim} Y=2, \operatorname{dim} Z=4 ; \Lambda_{*}$ has the topology defined by $\nu$. 
The following assertions are clear:

(a) In $\Lambda_{2 n}:\left(R_{p}\right) \rightarrow 0$ iff $\nu\left(R_{p}\right) \rightarrow \infty$.

(b) If $P(X, Y, Z) \in \Lambda_{2 m+2 n}, Q(X, Y, Z) \in \Lambda_{2 n}$ and $\left(R_{p}\right)$ a sequence in $\Lambda_{2 m}$ such that $R_{p} \rightarrow R$ and $\nu\left(P-R_{p} Q\right) \rightarrow \infty$ then $R Q=P$.

(c) If $\nu\left(R_{p}\right) \rightarrow \infty$ then the sequence $\left(M_{p}\right)$ defined by $M_{p}=R_{0}+$ $\cdots+R_{p}$ converges to a unique limit denoted by $\sum_{p \geq 0} R_{p}$.

In Sections II and III we shall define three elements $A_{k} \in \tilde{U}^{2}\left(B \Gamma_{k}\right)$, $B_{k} \in \tilde{U}^{2}\left(B \Gamma_{k}\right), D_{k} \in \tilde{U}^{4}\left(B \Gamma_{k}\right)$; as the $U^{*}$-AHSS for $B \Gamma_{k}$ collapses there is a unique continuous homomorphism $\varphi$ of graded $U^{*}(p t)$ algebras: $\Lambda_{*} \rightarrow U^{*}\left(B \Gamma_{k}\right)$ such that $\varphi(X)=A_{k}, \varphi(Y)=B_{k}, \varphi(Z)=$ $D_{k}$.

The next well known result will be useful:

Proposition 1.2. Suppose $X$ a $C W$-complex such that $H^{*}(X)=$ $\mathbb{Z}[a]$. Then there is an element $A \in U^{*}(X)$ such that $\mu(A)=a$ and $U^{*}(X)=H^{*}(X) \hat{\otimes} U^{*}(p t)=U^{*}(p t)[[A]]$. Moreover for any $A^{\prime} \in U^{*}(X)$ such that $\mu\left(A^{\prime}\right)=a$ we have $U^{*}(X)=U^{*}(p t)\left[\left[A^{\prime}\right]\right]$.

II. Computation of $U^{*}(B \Gamma)$. We recall that the quaternion group $\Gamma$ consists of $\{1, \pm i, \pm j, \pm k\}$ subject to the relations $i j=k, j k=i$, $k i=j, i^{2}=k^{2}=-1$. The irreducible unitary representations of $\Gamma$ are $1: i \rightarrow 1, j \rightarrow 1, \xi_{i}: i \rightarrow 1, j \rightarrow-1, \xi_{j}: i \rightarrow-1, j \rightarrow 1, \xi_{k}: i \rightarrow-1$, $j \rightarrow-1, \eta: i \rightarrow\left(\begin{array}{cc}i & 0 \\ 0 & -i\end{array}\right), j \rightarrow\left(\begin{array}{cc}0 & -1 \\ 1 & 0\end{array}\right)$; the character table of $\Gamma$ is:

(Conjugacy classes)

\begin{tabular}{c|c|c|c|c|c|} 
& 1 & -1 & $\pm i$ & $\pm j$ & $\pm k$ \\
\hline 1 & 1 & 1 & 1 & 1 & 1 \\
\hline$\xi_{i}$ & 1 & 1 & 1 & -1 & -1 \\
\hline$\xi_{j}$ & 1 & 1 & -1 & 1 & -1 \\
\hline$\xi_{k}$ & 1 & 1 & -1 & -1 & 1 \\
\hline$\eta$ & 2 & -2 & 0 & 0 & 0
\end{tabular}

We have the following relations in the representation ring $R(\Gamma)$ :

$$
\begin{array}{cl}
\xi_{i}^{2}=\xi_{j}^{2}=\xi_{k}^{2}=1, & \xi_{i} \cdot \xi_{j}=\xi_{k}, \quad \xi_{j} \cdot \xi_{k}=\xi_{i}, \quad \xi_{k} \xi_{i}=\xi_{j}, \\
\eta \cdot \xi_{i}=\eta \cdot \xi_{j}=\eta, & \eta^{2}=1+\xi_{i}+\xi_{j}+\xi_{k} \quad \text { (see [6], [2]). }
\end{array}
$$


We have $H^{0}(B \Gamma)=\mathbb{Z}, H^{4 n}(B \Gamma)=\mathbb{Z}_{8}, n \geq 1, H^{4 n+2}(B \Gamma)=\mathbb{Z}_{2} \oplus \mathbb{Z}_{2}$, $n \geq 0, H^{2 n+1}(B \Gamma)=0$. Moreover if $d$ is a generator of $H^{4}(B \Gamma)$ and if $a, b$ are generators of $H^{2}(B \Gamma)$ then $d^{n}$ is a generator of $H^{4 n}(B \Gamma)$, $n \geq 1$, and $a d^{n}, b d^{n}$ are generators of $H^{4 n+2}(B \Gamma), n \geq 0$ (see [5]).

Since $H^{m}(B \Gamma)=0, m$ odd we have:

Proposition 2.1. The $U^{*}-A H S S$ for $B \Gamma$ collapses.

There are four important complex vector-bundles $\xi_{i}, \xi_{j}, \xi_{k}: E \Gamma \times_{\Gamma}$ $\mathbb{C} \rightarrow B \Gamma$ and $\eta: E \Gamma \times_{\Gamma} \mathbb{C}^{2} \rightarrow B \Gamma$ where the actions of $\Gamma$ on $\mathbb{C}$ and $\mathbb{C}^{2}$ are induced by the representations $\xi_{i}, \xi_{j}, \xi_{k}$ and $\eta$. We have a canonical inclusion $q: \mathbb{Z}_{2} \subset \Gamma$ obtained by identifying $\left\{1, i^{2}\right\}$ with $\mathbb{Z}_{2}$; let $\rho$ be the unitary representation of $\mathbb{Z}_{2}$ given by $\rho(1)=1, \rho\left(i^{2}\right)=-1$; the restriction map: $R(\Gamma) \rightarrow R\left(\mathbb{Z}_{2}\right)$ sends $\xi_{i}, \xi_{j}, \xi_{k}$ to 1 and $\eta$ to $2 \rho$; so:

Proposition 2.2. $(B q)^{*}\left(\xi_{h}\right), h=i, j, k$, are trivial and $(B q)^{*}(\eta)=$ $2 \rho$.

1. Chern Classes of $\xi_{i}, \xi_{j}, \eta$. The canonical isomorphism

$$
\operatorname{Hom}(\Gamma, U(1)) \rightarrow H^{2}(\Gamma)
$$

is given by $\delta \rightarrow c_{1}(g(\delta))$ where $g$ denotes the canonical map: $R(\Gamma) \rightarrow$ $K^{0}(B \Gamma)$ and $c_{1}$ the first Chern class (Sec. [2]). Since $\operatorname{Hom}(\Gamma, U(1))=$ $\left\{1, \xi_{i}, \xi_{j}, \xi_{k}\right\}$ and $H^{2}(B \Gamma)=\mathbb{Z}_{2} \oplus \mathbb{Z}_{2}$ we have:

Proposition 2.3. $H^{2}(B \Gamma)$ is generated by $\left\{c_{1}\left(\xi_{i}\right), c_{1}\left(\xi_{j}\right)\right\}$.

Now we consider the topological group $\mathrm{Sp}(1)$ of quaternions of absolute value $1 ; \mathrm{Sp}(1)$ is homeomorphic to $S^{3}$ and $H^{*}\left(B S^{3}\right)=\mathbb{Z}[u]$, $\operatorname{dim} u=4, u$ being the first symplectic Pontrjagin class of the universal $\operatorname{Sp}(1)$-vector bundle $\theta$. If we consider $\theta$ as a $U(2)$-vector bundle, then $u=c_{2}(\theta)$ (see [12], page 179). Let $p: \Gamma \subset \operatorname{Sp}(1)=S^{3}$ be the natural inclusion; then it is easily seen that $(B p)^{*}(\theta)=\eta, \theta$ being regarded as a $U(2)$-vector bundle.

Proposition 2.4. We have $c_{1}(\eta)=0$ and $H^{4}(B \Gamma)$ is generated by $c_{2}(\eta)$

Proof. Since $\operatorname{det} \eta=1$ we have $c_{1}(\eta)=0$. From the transgression exact sequence of the fibration: $S^{3} / \Gamma \rightarrow B \Gamma \stackrel{B p}{\rightarrow} B S^{3}$ we get the exact 
sequence: $H^{4}\left(B S^{3}\right) \stackrel{(B p)^{*}}{\rightarrow} H^{4}(B \Gamma) \rightarrow H^{4}\left(S^{3} / \Gamma\right)=0$ and the result follows (see [11], page 519).

From 2.3, 2.4 we may take the Euler classes $e_{1}(\eta)=d$ as a generator of $H^{4}(B \Gamma)$ and $\left\{a=e_{1}\left(\xi_{i}\right), b=e_{1}\left(\xi_{j}\right)\right\}$ as a system of generators of $H^{2}(B \Gamma)$. Moreover $e_{1}(n \cdot \eta)=e_{1}(\eta)^{n}=d^{n}$ and $\left\{e_{1}\left(\xi_{i}+n \cdot \eta\right)=a d^{n}\right.$, $\left.e_{1}\left(\xi_{j}+n \cdot \eta\right)=b d^{n}\right\}$ are generators of $H^{4 n}(B \Gamma), n \geq 1$ and $H^{4 n+2}(B \Gamma)$, $n \geq 0$, respectively.

2. Computation of $U^{*}(B \Gamma)$. Let $A, B, D$ be the Euler classes for $M U$ of $\xi_{i}, \xi_{j}, \eta: e\left(\xi_{i}\right)=A \in \tilde{U}^{2}(B \Gamma), e\left(\xi_{j}\right)=B \in \tilde{U}^{2}(B \Gamma), e(\eta)=$ $D \in \tilde{U}^{4}(B \Gamma)$. We recall that $\Lambda_{*}=U^{*}(p t)[[X, Y, Z]]$ is graded by taking $\operatorname{dim} X=\operatorname{dim} Y=2, \operatorname{dim} Z=4$; there is a unique continuous homomorphism $\varphi: \Lambda_{*} \rightarrow U^{*}(B \Gamma)$ of graded $U^{*}(p t)$-algebras such that $\varphi(X)=A, \varphi(Y)=B, \varphi(Z)=D$. In particular if $P(Z)=\alpha_{0}+\alpha_{1} Z+$ $\cdots+\alpha_{i} Z^{i}+\cdots \in \Lambda_{2 n}$ then $\varphi(P)=P(D)=\operatorname{Lim}_{n \rightarrow \infty}\left(\alpha_{0}+\cdots+\alpha_{n} \cdot D^{n}\right)$ in $U^{2 n}(B \Gamma)$. If $U^{*}(p t)[[D]]=\left\{R(D), R(Z) \in \Omega_{*}\right\}$, then $U^{*}(p t)[[D]]$ is a sub- $U^{*}(p t)$-algebra of $U^{*}(B \Gamma)$.

THEOREM 2.5. $U^{*}(B \Gamma)$ is concentrated in even dimensions and as a $U^{*}(p t)[[D]]-$ module $U^{*}(B \Gamma)$ is generated by $1, A, B$.

Proof. We have $U^{2 n+1}(B \Gamma)=0$ because $J^{p, q}=J^{p+1, q-1}$ if $p+q=$ $2 n+1$ and then $U^{2 n+1}(B \Gamma)=J^{0,2 n+1}=\bigcap_{p+q=2 n+1} J^{p, q}=0$ (see Section I).

Suppose $2 n=4 m+2>0$. If $x \in U^{4 m+2}(B \Gamma)=J^{0,4 m+2}=J^{4 m+2,0}$ then $\mu(x)=\alpha_{m} a d^{m}+\beta_{m} b d^{m}=\mu\left(\alpha_{m} A D^{m}+\beta_{m} B D^{m}\right), \alpha_{m} \in U^{0}(p t)=$ $\mathbb{Z}, \beta_{m} \in U^{0}(p t)=\mathbb{Z}$. It follows that $\mu\left(x-\left(\alpha_{m} A D^{m}+\beta_{m} B D^{m}\right)\right)=0$ and $x_{1}=x-\left(\alpha_{m} A D^{m}+\beta_{m} B D^{m}\right) \in J^{4 m+3,-1}=J^{4 m+4,-2}$. Let $s_{1}$ be the quotient map: $J^{4 m+4,-2} \rightarrow J^{4 m+4,-2} / J^{4 m+5,-3}=H^{4 m+4}\left(B \Gamma, U^{-2}(p t)\right)$ $=U^{-2}(p t) \otimes H^{4 m+4}(B \Gamma)$. Then $s_{1}\left(x_{1}\right)=\gamma_{m+1} \otimes d^{m+1}, \gamma_{m+1} \in U^{-2}(p t)$. From the following commutative diagram where $\chi$ is induced by the $U^{*}(p t)$-module-structure:

$$
\begin{array}{lllll}
U^{-2}(p t) \otimes U^{4 m+4}(B \Gamma)=U^{-2}(p t) \otimes J^{4 m+4,0} & \stackrel{\chi}{\rightarrow} & J^{4 m+4,-2} \\
1 \otimes \mu & \downarrow & & & \downarrow s_{1} \\
U^{-2}(p t) & \otimes H^{4 m+4}(B \Gamma) & \stackrel{\sim}{\longrightarrow} & H^{4 m+4}\left(B \Gamma, U^{-2}(p t)\right)
\end{array}
$$

it follows that $s_{1}\left(x_{1}\right)=s_{1}\left(\gamma_{m+1} D^{m+1}\right)$ and then $s_{1}\left(x_{1}-\gamma_{m+1} D^{m+1}\right)=0$; so $\left(x_{1}-\gamma_{m+1}\right) D^{m+1} \in J^{4 m+5-3}=J^{4(m+1)+2,-4}$. We have $x_{2}=x_{1}-$ $\gamma_{m+1} D^{m+1}=x-\left(A \cdot \alpha_{m} D^{m}+B \cdot \beta_{m} D^{m}+\gamma_{m+1} D^{m+1}\right) \in J^{4(m+1)+2,-4}$. 
By using again the products $\chi$ we see that after a finite number of steps there are three polynomials in $Z$ :

$$
\begin{gathered}
P_{q}(Z)=\alpha_{m} Z^{m}+\alpha_{m+1} Z^{m+1}+\cdots+\alpha_{m+q-1} Z^{m+q-1}, \\
Q_{q}(Z)=\beta_{m} Z^{m}+\beta_{m+1} Z^{m+1}+\cdots+\beta_{m+q-1} Z^{m+q-1}, \\
R_{q}(Z)=\gamma_{m+1} Z^{m+1}+\cdots+\gamma_{m+q} Z^{m+q}, \quad \text { with } \\
\operatorname{deg} P_{q}=m+(q-1), \quad \operatorname{deg} Q_{q}=m+(q-1), \\
\operatorname{deg} R_{q}=m+q \text { such that } \\
\text { (1) } x-\left(A \cdot P_{q}(D)+B Q_{q}(D)+R_{q}(D)\right) \in J^{4(m+q)+2,-4 q}
\end{gathered}
$$

Furthermore

$$
\begin{aligned}
& P_{q+1}(Z)=P_{q}(Z)+\alpha_{m+q} Z^{m+q} \\
& Q_{q+1}(Z)=Q_{q}(Z)+\beta_{m+q} Z^{m+q} \\
& R_{q+1}(Z)=R_{q}(Z)+\gamma_{m+q+1} Z^{m+q+1}
\end{aligned}
$$

If

$$
\begin{aligned}
& P(Z)=\sum_{i=m}^{\infty} \alpha_{i} Z^{i} \in \Lambda_{4 m} \\
& Q(Z)=\sum_{i=m}^{\infty} \beta_{i} Z^{i} \in \Lambda_{4 m} \\
& R(Z)=\sum_{i=m+1}^{\infty} \gamma_{i} Z^{i} \in \Lambda_{4 m+2}
\end{aligned}
$$

then by using (1) and Section I we have $x=A P(D)+B Q(D)+R(D)$.

The cases $2 n=4 m+2<0$ and $2 n=4 m$ are similar.

The next two propositions will be used later on.

Proposition 2.6. If

$$
H(Z)=\sum_{i=0} \alpha_{i} Z^{i} \in \Lambda_{2 n}
$$

is such that $H(D)=0$, then $\alpha_{0}=0$ and if $\alpha_{p}$ is the leading coefficient, we have $\alpha_{p} \in 8 \cdot U^{*}(p t)$.

Proof. Since $D \in \tilde{U}^{*}(B \Gamma)$ we have

$$
\sum_{i=1}^{\infty} \alpha_{i} D^{i}=D\left(\sum_{i=1}^{\infty} \alpha_{i} D^{i-1}\right) \in \widetilde{U^{*}}(B \Gamma) ;
$$

then $\alpha_{0} \cdot 1 \in \tilde{U}^{*}(B \Gamma) \cap U^{*}(p t)=\{0\}$ and $\alpha_{0} \cdot 1=0$. If $i$ denotes the inclusion $\{*\} \subset B \Gamma$ we have $i^{*}\left(\alpha_{0} \cdot 1\right)=\alpha_{0}=0$. Then $H(Z)=$ 
$\alpha_{p} Z^{p}+\cdots+\alpha_{m} Z^{m}+\cdots, \alpha_{p} \neq 0, p \geq 1$. From $\alpha_{q} D^{q} \in J^{4 q, 2 n-4 q} \subset$ $J^{4 p+4,2 n-(4 p+4)}, q \geq p+1$, it follows that $t_{q}=\alpha_{p+1} D^{p+2}+\cdots+\alpha_{q} D^{q} \in$ $J^{4 p+4,2 n-(4 p+4)}, q \geq p+1$. Since $J^{4 p+4,2 n-(4 p+4)}$ is closed for the topology $T$ of $U^{2 n}(B \Gamma)$ we have

$$
\sum_{i=p+1}^{\infty} \alpha_{i} D^{i} \in J^{4 p+4,2 n-(4 p+4)} \subset J^{4 p+1,2 n-(4 p+1)} .
$$

Let $s$ be the quotient map

$$
\begin{aligned}
& J^{4 p, 2 n-4 p} \rightarrow J^{4 p, 2 n-4 p} / J^{4 p+1,2 n-(4 p+1)} \\
& \quad=H^{4 p}\left(B \Gamma, U^{2 n-4 p}(p t)\right)=H^{4 p}(B \Gamma) \otimes U^{2 n-4 p}(p t) \\
& \quad=\mathbb{Z}_{8} \otimes U^{2 n-4 p}(p t)=U^{2 n-4 p}(p t) / 8 \cdot U^{2 n-4 p}(p t) .
\end{aligned}
$$

Then:

$$
0=s(H(D))=s\left(\alpha_{p} D^{p}\right)+s\left(\sum_{i=p+1} \alpha_{i} D^{i}\right)=s\left(\alpha_{p} D^{p}\right)=\alpha_{p} \otimes d^{p} ;
$$

since $d^{p}$ is a generator of $H^{4 p}(B \Gamma)$ we have $\alpha_{p} \in 8 U^{2 n-4}(p t)$.

Let $F$ be the formal group law and [2] $(Y)=F(Y, Y)$; if $\rho$ is the nontrivial unitary irreducible representation for $\mathbb{Z}_{2}$ then we get (see [9]):

Proposition 2.7. $U^{*}\left(B \mathbb{Z}_{2}\right)=U^{*}(p t)[[Y]] /([2](Y))$ and the image of $Y$ by the quotient map: $U^{*}(p t)[[Y]] \rightarrow U^{*}\left(B \mathbb{Z}_{2}\right)$ is the Euler class $e(\rho)$.

We have adopted the following graduation in 2.7: if

$$
F(X, Y)=X+Y+a_{11} X Y+\sum_{i \geq 1, j \geq 1} a_{i j} X^{i} Y^{j},
$$

then $\left|a_{i j}\right|=2(1-i-j),|X|=|Y|=2$; so $F(X, Y) \in \Lambda_{2}$. We shall often make use of the coefficient $a_{11}$. We know that there is a unique formal power series $[-1](Y) \in U^{*}(p t)[[Y]]\left(\subset \Lambda_{2}\right)$ such that: $F(Y,[-1](Y))$ $=0$.

Proposition 2.8. There is $P_{0}(Z) \in \Omega_{2}, P_{0}(Z)=b_{1} Z+\sum_{i \geq 1} b_{i} Z^{i}$ such that $c f_{1}(\eta)=P_{0}(D)$. The coefficients $b_{i}, i \geq 1$, are determined by the relation $\sum_{i \geq 1} b_{i}(Y \cdot[-1](Y))^{i}=Y+[-1] Y$; in particular $b_{1}=-a_{11}$.

Proof. We have seen that if $\theta$ is the universal $S p(1)$-bundle over $S p(1)=B S^{3}$ considered as a $U(2)$-vector bundle then $\eta=(B p)^{*}(\theta)$, 
$p: \Gamma \subset S p(1)$. As $H^{*}\left(B S^{3}\right)=\mathbb{Z}[u], u=c_{2}(\theta)$, we have $U^{*}\left(B S^{3}\right)=$ $U^{*}(p t)[[V]], V=e(\theta)$, the Euler class of $\theta$ for $M U$. Hence there is $P_{0}(Z)=\sum_{i \geq 1} b_{i} Z^{i} \in \Omega_{2}$ such that $P_{0}(V)=c f_{1}(\theta)$; it follows that

$$
c f_{1}(\eta)=(B p)^{*}\left(c f_{1}(\theta)\right)=(B p)^{*}\left(\sum_{i \geq 1} b_{i} V^{i}\right)=\sum_{i \geq 1} b_{i} D^{i}=P_{0}(D) .
$$

The relation $\sum_{i \geq 1} b_{i}(Y \cdot[-1] Y)^{i}=Y+[-1](Y)$ is proved in the Appendix part $\mathrm{B}$ and gives $b_{1}=-a_{11}$.

We recall that $A=c f_{1}\left(\xi_{i}\right) \in \tilde{U}^{2}(B \Gamma), B=c f_{1}\left(\xi_{j}\right) \in \tilde{U}^{2}(B \Gamma), D=$ $c f_{2}(\eta) \in \tilde{U}^{4}(B \Gamma)$; let $C \in \tilde{U}^{2}(B \Gamma)$ be $c f_{1}\left(\xi_{k}\right)$.

Proposition 2.9. (a) There are $P(Z) \in \Omega_{2}, Q(Z) \in \Omega_{4}, P(Z)=$ $-4 a_{11} Z+\sum_{i \geq 2} \alpha_{i} Z^{i}, Q(Z)=4 Z+\sum_{i \geq 2} \beta_{i} Z^{r}, \beta_{2} \notin 2 U^{*}(p t)$, such that $c f_{1}\left(\eta^{2}\right)=P(D)=A+B+C, c f_{2}\left(\eta^{2}\right)=Q(D)=A B+B C+C A$.

(b) $c f_{3}\left(\eta^{2}\right)=A B C=0$,

(c) $A^{3}=-A Q(D)+A^{2} P(D), B^{3}=-B Q(D)+B^{2} P(D)$.

Proof. (a) Let $g: B \Gamma \rightarrow B U(2)$ be a map classifying $\eta$; then $\eta^{2}$ is classified by the composite: $B \Gamma \stackrel{\Delta}{\rightarrow} B \Gamma \times B \Gamma \stackrel{g \times g}{\rightarrow} B U(2) \times B U(2) \stackrel{m}{\rightarrow}$ $B U(4)$, where $m$ is a map classifying $\gamma(2) \otimes \gamma(2)$ and $\Delta$ the diagonal map. We have $U^{*}(B U(2) \times B U(2))=U^{*}(p t)\left[\left[c_{1}, c_{2}, c_{1}^{\prime}, c_{2}^{\prime}\right]\right], c_{1}$, $c_{2}, c_{1}^{\prime}, c_{2}^{\prime}$ being respectively the images of $c f_{1}(\gamma(2)) \otimes 1, c f_{2}(\gamma(2)) \otimes$ $1,1 \otimes c f_{1}(\gamma(2)), 1 \otimes c f_{2}(\gamma(2))$ by the canonical map: $U^{*}(B U(2)) \otimes$ $U^{*}(B U(2)) \stackrel{X}{\rightarrow} U^{*}(B U(2) \times B U(2))$. Since the following diagram commutes:

$$
\begin{array}{ccc}
U^{*}(B U(4)) \stackrel{m^{*}}{\rightarrow} U^{*}(B U(2) \times B U(2)) & \stackrel{(g \times g)^{*}}{\rightarrow} & U^{*}(B \Gamma \times B \Gamma) \stackrel{\Delta^{*}}{\rightarrow} U^{*}(B \Gamma) \\
X \uparrow & & \uparrow \nearrow \cup \\
U^{*}(B U(2)) \otimes B^{*}(B U(2)) & \stackrel{X}{\rightarrow} & U^{*}(B \Gamma) \otimes U^{*}(B \Gamma)
\end{array}
$$

we must substitute $c f_{1}(\eta)$ for $c_{1}, c_{1}^{\prime}, c f_{2}(\eta)$ for $c_{2}, c_{2}^{\prime}$ in $m^{*}\left(c f_{1}(\gamma(4))\right)$, $m^{*}\left(c f_{2}(\gamma(4))\right), m^{*}\left(c f_{3}(\gamma(4))\right)$ in order to calculate $c f_{1}\left(\eta^{2}\right), c f_{2}\left(\eta^{2}\right)$, $c f_{3}\left(\eta^{2}\right)$ (see Sec. I).

We have $m^{*}\left(c f_{1} \gamma(4)\right)=\sum a_{(u, v)} c_{1}^{u_{1}} c_{2}^{u_{2}} c_{1}^{\prime} v_{1} c_{2}^{\prime v_{2}}, u=\left(u_{1}, u_{2}\right), v=$ $\left(v_{1}, v_{2}\right), u_{1} \geq 0, u_{2} \geq 0, v_{1} \geq 0, v_{2} \geq 0$. It is important to calculate $a_{(u, v)}$ when $u_{1}=u_{2}=0$, or $v_{1}=v_{2}=0$.

Suppose $u_{1}=u_{2}=0$. We denote by 0 the pair $(0,0)$. Then the coefficients $a_{(0, v)}$ are given by $i^{*} \circ m^{*}\left(c f_{1}(\gamma(4))\right), i$ being the natural inclusion:

$$
\{*\} \times B U(2) \stackrel{i}{\rightarrow} B U(2) \times B U(2)
$$


Since $i^{*} \circ m^{*}(\gamma(4))=\gamma(2)+\gamma(2)$ we have $i^{*} \circ m^{*}\left(c f_{1}(\gamma(4))\right)=2 c_{1}^{\prime}$. Similarly $a_{(u, 0)}=2 c_{1}$. Hence

$$
m^{*}\left(c f_{1}(\gamma(4))\right)=2\left(c_{1}+c_{1}^{\prime}\right)+\sum_{\substack{\|u\| \geq 1 \\\|v\| \geq 1}} a_{(u, v)} c_{1}^{u_{1}} c_{2}^{u_{2}} c_{1}^{\prime} v_{1} c_{2}^{\prime} v_{2}
$$

where $\|u\|=u_{1}+u_{2},\|v\|=v_{1}+v_{2}$.

We recall that $c f_{1}(\eta)=P_{0}(D), P_{0}(Z) \in \Omega_{2}, \nu^{\prime}\left(P_{0}\right)=1, \nu^{\prime}=\frac{1}{4} \nu$ (see Sec. I). Consider

$$
\begin{aligned}
P(Z) & =2\left(P_{0}(Z)+P_{0}(Z)\right)+\sum_{\substack{\|u\| \geq 1 \\
\|v\| \geq 1}} a_{(u, v)} P_{0}^{u_{1}+v_{1}}(Z) Z^{u_{2}+v_{2}} \\
& =4 b_{1} Z+\sum_{i \geq 2} \alpha_{i} Z^{i},
\end{aligned}
$$

$b_{1}$ being the first coefficient $\neq 0$ of $P_{0}(Z)$ because $u_{1}+v_{1}+u_{2}+v_{2} \geq 2$ when $\|u\| \geq 1,\|v\| \geq 1$. Hence $c f_{1}\left(\eta^{2}\right)=P(D)$. We remark that $P(Z) \in \Omega_{2}$.

There are unique elements $b_{(u, v)} \in U^{*}(p t)$ such that $m^{*}\left(c f_{2}(\gamma(4))\right)=$ $\sum b_{(u, v)} c_{1}^{u_{1}} c_{2}^{u_{2}} c_{1}^{\prime v_{1}} c_{2}^{\prime v_{2}}$. Then the coefficients $b_{(u, 0)}$ and $b_{(0, v)}$ are given by $c f_{2}(\gamma(2)+\gamma(2))=c f_{1}^{2}(\gamma(2))+2 c f_{2}(\gamma(2))$. Hence

$$
m^{*}\left(c f_{2}(\gamma(4))=c_{1}^{2}+c_{1}^{2}+2\left(c_{2}+c_{2}^{1}\right)+\sum_{\|u\| \geq 1,\|v\| \geq 1} b_{u, v} c_{1}^{u_{1}} c_{2}^{u_{2}} c_{1}^{\prime v_{1}} c_{2}^{\prime v_{2}} .\right.
$$

Consider

$$
\begin{aligned}
Q(Z) & =4 Z+2 P_{0}^{2}(Z)+\sum_{\|u\| \geq 1,\|v\| \geq 1} b_{(u, v)} P_{0}^{u_{1}+v_{1}}(Z) Z^{u_{2}+v_{2}} \\
& =4 Z+\sum_{i \geq 2} \beta_{i} Z^{i} .
\end{aligned}
$$

Then $c f_{2}\left(\eta^{2}\right)=Q(D), Q(Z) \in \Omega_{4}$.

Let $q$ be the inclusion $\mathbb{Z}_{2} \subset \Gamma$; since $(B q)^{*}\left(\xi_{h}\right), h=i, j, k$, are trivial by 2.2 we have $(B q)^{*}(A)=(B q)^{*}(B)=(B q)^{*}(C)=0$ and since $Q(D)=c f_{2}\left(\eta^{2}\right)=A B+B C+C A$ we have $(B q)^{*}(Q(D))=0$. It follows by 2.7 that $(B q)^{*}(D)=d^{2}, d$ being the image of $Y$ by the quotient map:

$$
U^{*}(p t)[[Y]] \rightarrow U^{*}(p t)[[Y]] /([2](Y)) .
$$

Thus:

$$
\begin{aligned}
4 Y^{2} & +\sum_{i \geq 2} \beta_{i} \cdot Y^{2 i}=[2](Y) \cdot G(Y) \\
& =\left(2 Y+a_{11} Y^{2}+a_{3} Y^{3}+\cdots\right)\left(\varepsilon_{0} Y+\varepsilon_{1} Y^{2}+\varepsilon_{2} Y^{3}+\cdots\right) \text { and }
\end{aligned}
$$




$$
\begin{aligned}
& \varepsilon_{0}=2, \quad 0=2 \varepsilon_{1}+a_{11} \varepsilon_{0}=2\left(\varepsilon_{1}+a_{11}\right) ; \quad \text { so } \\
& \varepsilon_{1}=-a_{11}, \quad \beta_{2}=2 \varepsilon_{2}-a_{11}^{2}+2 a_{3} ;
\end{aligned}
$$

since $a_{11}^{2} \notin 2 U^{*}(p t)$ (because $\left.U^{*}(p t)=\left[x_{1}, x_{2}, \ldots\right], a_{11}=-x_{1}\right)$ it follows that $\beta_{2} \notin 2 U^{*}(p t)$. The relations $P(D)=A+B+C, Q(D)=$ $A B+B C+C A$ are easy consequences of the relation $\eta^{2}=1+\xi_{i}+\xi_{j}+\xi_{k}$.

(b) The above relation gives $c f_{3}\left(\eta^{2}\right)=A B C$; in order to show that $A B C=0$ we consider the Boardman map $B d: U^{*}(B \Gamma) \rightarrow$ $K^{*}(B \Gamma) \hat{\otimes} \mathbb{Z}\left[a_{1}, a_{2}, \ldots\right]$ (see [8], page 358). This map is a ring-homomorphism which is injective because $B \Gamma$ has a periodic cohomology; furthermore if $\tau$ is a line complex vector bundle over $B \Gamma$ we have:

$$
B d(e(\tau))=(\tau-1)+(\tau-1)^{2} \otimes a_{1}+(\tau-1)^{3} \otimes a_{2}+\cdots ;
$$

as $\left(\xi_{i}-1\right)\left(\xi_{j}-1\right)\left(\xi_{k}-1\right)=0$ we get $B d(A B C)=0$ and $A B C=0$.

(c) We have $Q(D)=A(B+C)+B C=A(P(D)-A)+B C$; as $A B C=$ 0 we obtain $A^{3}=-A Q(D)+A^{2} P(D)$; similarly $B^{3}=-A Q(D)+$ $A^{2} P(D)$.

Proposition 2.10. There is $S(Z)=-a_{11} Z+\sum_{i \geq 2} s_{i} \cdot Z^{i} \in \Omega_{2}$ such that $A^{2}=A S(D), B^{2}=B S(D)$. Moreover:

$$
A B=(A+B)(P(D)-S(D))-Q(D),
$$

$P(Z), Q(Z)$ being as in 2.9 .

Proof. Consider the relation $\eta \xi_{i}=\eta$. If the vector bundle $\gamma(2) \otimes \gamma(1)$ over $B U(2) \times B U(1)$ is classified by $m_{1}: B U(2) \times B U(1) \rightarrow B U(2)$ and if $g: B \rightarrow B U(2), h: B \rightarrow B U(1)$ are classifying maps for $\eta$ and $\xi_{i}$, then $\eta \xi_{i}$ is classified by:

$$
B \Gamma \stackrel{\Delta}{\rightarrow} B \Gamma \times B \Gamma \stackrel{g \times h}{\rightarrow} B U(2) \times B U(1) \stackrel{m_{1}}{\rightarrow} B U(2) .
$$

We have the following commutative diagram:

$$
\begin{array}{ccc}
U^{*}(B U(2)) \stackrel{m_{\rightarrow}^{*}}{\rightarrow} U^{*}(B U(2)) \times B U(1) & \stackrel{(g \times h)^{*}}{\rightarrow} & U^{*}(B \Gamma \times B \Gamma) \stackrel{\Delta}{\rightarrow} U^{*}(B \Gamma) \\
X \uparrow & & X \uparrow \nearrow \text { cup-product } \\
U^{*}(B U(2)) \otimes U(B U(1)) & \stackrel{g^{*} \times h^{*}}{\rightarrow} & U^{*}(B \Gamma) \otimes U^{*}(B \Gamma) .
\end{array}
$$

Moreover $U^{*}(B U(2) \times B U(1))=U^{*}(p t)\left[\left[c_{1}, c_{2}, c_{1}^{\prime}\right]\right]$ where $c_{1}, c_{2}, c_{1}^{\prime}$ are the images respectively of $c f_{1} \gamma(2) \otimes 1, c f_{2} \gamma(2) \otimes 1,1 \otimes c f_{1} \gamma(1)$ by the canonical map: $U^{*}(B U(2)) \times U^{*}(B U(1)) \stackrel{X}{\rightarrow} U^{*}(B U(2) \times B U(1))$. Then

$$
m_{1}^{*}\left(c f_{2}(\gamma(2))\right)=\sum e_{(u, v)} c_{1}^{u_{1}} c_{2}^{u_{2}} c_{1}^{\prime v}, \quad u=\left(u_{1}, u_{2}\right)
$$


If $i$ and $j$ are the natural inclusions: $B U(2) \times\{*\} \rightarrow B U(2) \times B U(1)$ and $\{*\} \times B U(1) \rightarrow B U(2) \times B U(1)$, then the coefficients $e_{(u, 0)}$ and $e_{(0, v)}$ are given respectively by $i^{*} \circ m^{*}\left(c f_{2}(\gamma(2))\right)=c f_{2}(\gamma(2))=c_{2}$ and $j^{*} \circ m^{*}\left(c f_{2}(\gamma(2))\right)=c f_{2}(\gamma(1)+\gamma(1))=c_{1}^{\prime 2}$. Hence

$$
\begin{aligned}
m_{1}^{*}\left(c f_{2}(\gamma(2))\right)= & c_{2}+c_{1}^{\prime 2}+\sum_{\substack{\|u\| \geq 1 \\
v \geq 1}} e_{(u, v)} c_{1}^{u_{1}} c_{2}^{u_{2}} c_{1}^{\prime v} \\
= & c_{2}+c_{1}^{\prime 2}+c_{1}^{\prime} N_{1}\left(c_{1}, c_{2}\right)+c_{1}^{\prime 2} N_{2}\left(c_{1}, c_{2}\right) \\
& +\cdots+c_{1}^{\prime m} N_{m}\left(c_{1}, c_{2}\right)+\cdots .
\end{aligned}
$$

To calculate $c f_{2}\left(\eta \cdot \xi_{i}\right)$ we substitute $c f_{1}(\eta), c f_{2}(\eta), c f_{1}\left(\xi_{i}\right)$, respectively for $c_{1}, c_{2}, c_{1}^{\prime}$. We recall that $c f_{1}(\eta)=P_{0}(D), \nu^{\prime}\left(P_{0}\right)=1\left(\nu^{\prime}=\frac{1}{4} \nu\right.$; see Sec. I). We can substitute $P_{0}(Z)$ for $c_{1}$ and $Z$ for $c_{2}$ in $N_{m}\left(c_{1}, c_{2}\right)$ to obtain $M_{m}(Z) \in \Omega_{*}, \nu^{\prime}\left(M_{m}\right) \geq 1, m \geq 1$. We need to calculate the leading coefficient of $M_{1}(Z)$. To this purpose consider $T=B U(1) \times$ $B U(1)$ and $r: T \rightarrow B U(2)$ a map classifying $\pi_{1}^{*}(\gamma(1))+\pi_{2}^{*}(\gamma(1)), \pi_{1}$, $\pi_{2}$ being respectively the first and second projections $T \rightarrow B U(1)$; we have $U^{*}(T \times B U(1))=U^{*}(p t)\left[\left[e_{1}, f_{1}, e_{1}^{\prime}\right]\right]$ with $(r \times 1){ }^{*}\left(c_{1}\right)=$ $e_{1}+f_{1},(r \times 1)^{*}\left(c_{2}\right)=e_{1} f_{1},(r \times 1)^{*}\left(c_{1}^{\prime}\right)=e_{1}^{\prime}$; it is easily seen that $(r \times 1)^{*}\left(m_{1}^{*} c f_{2}(\gamma(2))\right)=F\left(e_{1}, e_{1}^{\prime}\right) F\left(f_{1}, e_{1}^{\prime}\right)$ where $F$ denotes the formal group law. It follows that $e_{((1,0), 1)}=1, e_{((0,1), 1)}=2 a_{11}$ and $M_{1}(Z)=$ $a_{11} Z+\sum_{i \geq 2} b_{i}^{\prime} Z^{i}, \nu^{\prime}\left(M_{1}\right)=1$.

Now from the relation $A^{3}=-A Q(D)+A^{2} P(D)$ we deduce that $A^{n}=A Q_{n}(D)+A^{2} P_{n}(D), n \geq 3$, with $Q_{n}(Z) \in \Omega_{2 n-2}, P_{n}(Z) \in$ $\Omega_{2 n-4}, Q_{3}(Z)=-Q(Z), P_{3}(Z)=P(Z), Q_{n+1}(Z)=-Q(Z) P_{n}(Z)$, $P_{n+1}(Z)=P(Z) P_{n}(Z)+Q_{n}(Z)$. Then $\nu^{\prime}\left(P_{n+1}\right) \geq \inf \left(\nu^{\prime}\left(P_{n}\right), \nu^{\prime}\left(P_{n-1}\right)\right)$ and $\nu^{\prime}\left(P_{n+1}\right) \geq(n+1) / 2$; so:

$$
\operatorname{Lim}_{n \rightarrow \infty} \nu^{\prime}\left(P_{n}\right)=\operatorname{Lim}_{n \rightarrow \infty} \nu^{\prime}\left(Q_{n}\right)=+\infty \text {. }
$$

Consider

$$
\begin{aligned}
M_{n}(X, Z)= & Z+X^{2}\left[1+M_{2}(Z)+P(Z) M_{3}(Z)+\cdots+P_{n}(Z) M_{n}(Z)\right] \\
& +X\left[M_{1}(Z)+Q_{3}(Z) M_{3}(Z)+\cdots+Q_{n}(Z) M_{n}(Z)\right] \in \Lambda_{4}
\end{aligned}
$$

As

$$
\operatorname{Lim}_{n \rightarrow \infty} \nu\left(P_{n} M_{n}\right)=\operatorname{Lim}_{n \rightarrow \infty} \nu\left(Q_{n} M_{n}\right)=+\infty
$$

it follows that $\operatorname{Lim}_{n \rightarrow \infty} M_{n}(X, Z)$ exists (see Sec. I) and may be written as: $Z+X^{2}[1+H(Z)]+X H_{1}(Z)$ with $H(Z) \in \Omega_{0}, \nu^{\prime}(H) \geq 1$. We remark that the leading coefficient of $H_{1}(Z)$ is that of $M_{1}(Z)$; so: $H_{1}(Z)=a_{11} Z+\sum_{i \geq 2} d_{i} Z^{i} \in \Omega_{2}$. Thus: $c f_{2}\left(\eta \xi_{i}\right)=D+$ $A^{2}[1+H(D)]+A H_{1}(D)=c f_{2}(\eta)=D$ and $A^{2}[1+H(D)]=$ 
$-A H_{1}(D)$. Let $E(Z) \in \Omega_{0}$ be such that $E(Z)(1+H(Z))=1$; hence $A^{2}=A S(D)$ with $S(Z)=-H_{1}(Z) E(Z)=-a_{11} Z+\sum_{i \geq 2} s_{i} Z^{i} \in \Omega_{2}$. Similarly $B^{2}=B S(D)$. Now

$$
\begin{aligned}
A B & =A B+B C+C A-C(A+B) \\
& =Q(D)-[P(D)-(A+B)] \cdot(A+B) \\
& =Q(D)-P(D) \cdot(A+B)+2 A B+(A+B) S(D) \\
& =2 A B+Q(D)+(A+B)(S(D)-P(D)) .
\end{aligned}
$$

Then:

$$
A B=(A+B)[P(D)-S(D)]-Q(D) .
$$

LEMMA 2.11. There is $T(Z)=8 Z+2 \lambda_{2} Z^{2}+\sum_{i \geq 3} \lambda_{i} Z^{i} \in \Omega_{4}, \lambda_{2} \notin$ $2 U^{*}(p t)$ and $T(D)=0$.

Proof. From $\eta^{2}=1+\xi_{i}+\xi_{j}+\xi_{k}$ we get $\eta^{3}=4 \eta$. Let $g_{1}: B \Gamma \rightarrow$ $B U(4)$ and $g: B \Gamma \rightarrow B U(2)$ be classifying maps (respectively) for $\eta^{2}$ and $\eta$; then $\eta^{3}$ is classified by: $B \Gamma \stackrel{\Delta}{\rightarrow} B \Gamma \times B \Gamma \stackrel{g_{1} \times g}{\rightarrow} B U(4) \times$ $B U(2) \stackrel{m_{2}}{\rightarrow} B U(8), m_{2}$ being a map classifying $\gamma(4) \otimes \gamma(2)$. Then we get $m_{2}^{*}\left(c f_{2}(\gamma(8))\right)=\sum f_{(u, v)} c_{1}^{u_{1}} c_{2}^{u_{2}} c_{3}^{u_{3}} c_{4}^{u_{4}} c_{1}^{\prime} v_{1} c_{2}^{\prime v_{2}}$, with $u=\left(u_{1}, u_{2}, u_{3}, u_{4}\right)$, $v=\left(v_{1}, v_{2}\right)$. The coefficients $f_{(u, 0)}$ and $f_{(0, v)}$ are given respectively by $c f_{2}(\gamma(4)+\gamma(4))=c_{1}^{2}+2 c_{2}$ and $c f_{2}(4 \gamma(2))=6 c_{1}^{\prime 2}+4 c_{2}^{\prime}$. Thus

$$
\begin{aligned}
m_{2}^{*}\left(c f_{2}(\gamma(8))\right)= & c_{1}^{2}+2 c_{2}+6 c_{1}^{\prime 2}+4 c_{2}^{\prime} \\
& +\sum_{\substack{\|u\| \geq 1 \\
\|v\| \geq 1}} f_{(u, v)} c_{1}^{u_{1}} c_{2}^{u_{2}} c_{3}^{u_{3}} c_{4}^{u_{4}} c_{1}^{\prime} v_{1} c_{2}^{\prime v_{2}} .
\end{aligned}
$$

In order to calculate $c f_{2}\left(\eta^{3}\right)$ we must substitute $c f_{1}\left(\eta^{2}\right)=P(D)$, $c f_{2}\left(\eta^{2}\right)=Q(D), c f_{3}\left(\eta^{2}\right)=0, c f_{4}\left(\eta^{2}\right)=0, c f_{1}(\eta)=P_{0}(D), c f_{2}(\eta)=D$ respectively for $c_{1}, c_{2}, c_{3}, c_{4}, c_{1}^{\prime}, c_{2}^{\prime}$. Consider

$$
\begin{aligned}
E(Z)= & P^{2}(Z)+2 Q(Z)+6 P_{0}^{2}(Z)+4 Z \\
& +\sum_{\|u\| \geq 1,\|v\| \geq 1} f_{(u, v)} P^{u_{1}}(Z) Q^{u_{2}}(Z) P_{0}^{v_{1}}(Z) \cdot Z^{v_{2}},
\end{aligned}
$$

$u=\left(u_{1}, u_{2}, 0,0\right), v=\left(v_{1}, v_{2}\right)$. Hence $E(D)=c f_{2}\left(\eta^{3}\right)$; but as the leading coefficients of $P(Z)$ and $Q(Z)$ belong to $4 U^{*}(p t), E(Z)$ has the form: $2 Q(Z)+6 P_{0}^{2}(Z)+4 Z+4 \tau Z^{2}+\sum_{i \geq 3} \tau_{i} Z^{i}$. So: $E(D)=$ $2 Q(D)+6 P_{0}^{2}(D)+4 D+4 \tau D^{2}+\sum_{i \geq 3} \tau_{i} D^{i}=c f_{2}\left(\eta^{3}\right)=c f_{2}(4 \eta)=$ $6 c f_{1}^{2}(\eta)+4 c f_{2}(\eta)=6 P_{0}^{2}(D)+4 D$. Hence if $T(Z)=2 Q(Z)+4 \tau Z^{2}+$ $\sum_{i \geq 3} \tau_{i} Z^{i} \in \Omega_{4}$, then $T(D)=0$. As $Q(Z)=4 Z+\sum_{i \geq 2} \beta_{i} Z^{i}, \beta_{2} \notin$ $2 U^{*}(p t)$, we have: $T(D)=8 Z+2 \lambda_{2} Z^{2}+\sum_{i \geq 3} \lambda_{i} Z^{i}, \lambda_{2} \notin 2 U^{*}(p t)$. 
THEOREM 2.12. If $M(Z) \in \Omega_{*}$ is such that $M(D)=0$, then $M(Z) \in$ $\Omega_{*} T(Z)$.

Proof. We may suppose $M(Z) \in \Omega_{2 n}, n \in \mathbb{Z}$. If $M(Z)=\omega_{0}+$ $\sum_{i \geq 1} \omega_{i} Z^{i}$, then by 2.6 we have $\omega_{0}=0$ and the first coefficient $\omega_{i} \neq 0$, say $\omega_{P_{0}}$, is such that $P_{0} \geq 1, \omega_{p_{0}} \in 8 U^{*}(p t)$. Thus $M(Z)=8 \omega_{p_{0}}^{\prime} Z^{p_{0}}+$ $\sum_{i>P_{0}} \omega_{i} Z^{i}$. Consider $M_{1}(Z)=M(Z)-\omega_{P_{0}}^{\prime} \cdot Z^{P_{0}-1} \cdot T(Z) \in \Omega_{2 n}$. We have $\nu\left(M_{1}(Z)\right)>\nu(M(Z))$ and $M_{1}(D)=0$. Then $M_{1}(Z)=$ $8 \omega_{P_{1}}^{\prime} Z^{P_{1}}+\sum_{i>P_{1}} \theta_{i} \cdot Z^{i}, P_{1}>P_{0}$. We form

$$
M_{2}(Z)=M_{1}(Z)-\omega_{P_{1}}^{\prime} Z^{P_{1}-1} T(Z)
$$

and then $\nu\left(M_{2}\right)>\nu\left(M_{1}\right), M_{2}(D)=0$. After a finite number of steps we get $M_{r+1}(Z)=M(Z)-\left(\omega_{P_{0}}^{\prime} Z^{P_{0}-1}+\cdots+\omega_{P_{r}}^{\prime} Z^{P_{r}-1}\right) T(Z)$ such that $P_{r}>P_{r-1}>\cdots>P_{1}>P_{0}, \nu\left(M_{r_{1}}\right)>\nu\left(M_{r}\right)>\cdots>\nu\left(M_{1}\right)>\nu(M)$ and $M_{r+1}(D)=0$. Since $\operatorname{Lim}_{r \rightarrow \infty} \nu\left(M_{r}\right)=\infty$ it follows that $M(Z)=$ $\left(\sum_{k \geq 0} \omega_{P_{k}}^{\prime} \cdot Z^{p_{k}-1}\right) \cdot T(Z)$ (see Sec. I).

LeMmA 2.13. There is $J(Z)=\mu_{1} Z+\sum_{i \geq 2} \mu_{i} Z^{i} \in \Omega_{0}, \mu_{1} \notin 2 U^{*}(p t)$, such that $A[2+J(D)]=B[2+J(D)]=0$.

Proof. We have [2] $(Y)=2 Y+a_{11} Y^{2}+\sum_{i \geq 3} a_{i} Y^{i}$. As $\xi_{i}^{2}$ is trivial we have [2] $(A)=0$ and from $A^{2}=A S(D)\left(S(Z) \in \Omega_{2}\right)$ we get $A^{n}=$ $A S^{n-1}(D)$. Consider $H_{n}(X, Z)=X\left[2+a_{11} S(Z)+\cdots+a_{n} S^{n-1}(Z)\right]$. Since $\operatorname{Lim}_{n \rightarrow \infty} \nu\left(S^{n}\right)=\infty$ it follows that $\operatorname{Lim}_{n \rightarrow \infty} H_{n}(X, Z)$ exists and has the form $X[2+J(Z)]$, with

$$
J(Z)=a_{11} S(Z)+\sum_{n \geq 3} a_{n} S^{n-1}(Z)=-a_{11}^{2} Z+\sum_{i \geq 2} \mu_{i} Z^{i} .
$$

If $\mu_{1}=-a_{11}^{2}$ we see that $\mu_{1} \notin 2 U^{*}(p t)$. Thus $A(2+J(D))=[2](A)=0$. Similarly $B(2+J(D))=0$.

LEMMA 2.14. Suppose $X M(Z)+Y N(Z)+E(Z) \in \Omega_{*}$ is such that $A M(D)+B N(D)+E(D)=0$. Then the first coefficient $\neq 0$ of $M(Z)$ and the first coefficient $\neq 0$ of $N(Z)$ belong to $2 U^{*}(p t)$.

Proof. We may suppose $X M(Z) \in \Omega_{2 n}, Y N(Z) \in \Omega_{2 n}, E(Z) \in \Omega_{2 n}$, $n \in \mathbb{Z}$. We shall give a proof in the case: $0 \neq M(Z)=a_{p} Z^{p}+$ $a_{p+1} Z^{p+1}+\cdots, a_{p} \neq 0,0 \neq N(Z)=b_{q} Z^{q}+b_{q+1} Z^{q+1}+\cdots, b_{q} \neq 0$ and $p \leq q$. We observe that if $s \geq p$ then $A\left(a_{p} D^{p}+\cdots+a_{p+s} D^{p+s}\right) \in$ $J^{4 p+2,2 n-4 p-2}$ and consequently $A M(D) \in J^{4 p+2,2 n-4 p-2}$ because the subgroups $J^{*, *}$ are closed in $U^{*}(B \Gamma)$. Similarly

$$
A\left(a_{p+1} D^{p+1}+\cdots+a_{r} D^{r}+\cdots\right) \in J^{4 p+6,2 n-4 p-6}
$$


and consequently

$$
A\left(a_{p+1} D^{p+1}+\cdots+a_{r} D^{r}+\cdots\right) \in J^{4 p+3,2 n-4 p-3} .
$$

There are similar remarks concerning $B N(D)$. Since by hypothesis $p \leq q$ we have $4 p+2 \leq 4 q+2$ and $J^{4 p+2,2 n-4 p-2} \supset J^{4 q+2,2 n-4 q-2}$. We shall denote by $g$ the quotient map:

$$
\begin{array}{r}
J^{4 p+2,2 n-4 p-2} \rightarrow J^{4 p+2,2 n-4 p-2} / J^{4 p+3,2 n-4 p-3} \\
=\left[U^{h}(p t) / 2 U^{h}(p t)\right] \oplus\left[U^{h}(p t) / 2 U^{h}(p t)\right],
\end{array}
$$

with $h=2 n-4 p-2$. Then $g(A M(D))=\bar{a}_{p}, \bar{a}_{p}$ being the image of $a_{p}$ by the quotient map

$$
U^{h}(p t) \rightarrow U^{h}(p t) / 2 U^{h}(p t),
$$

$U^{h}(p t) / 2 U^{h}(p t)$ being the first summand.

(a) Suppose $E(D)=0$.

(i) $p=q$. We have $g(A M(D))=\bar{a}_{p}$ and $g(B M(D))=\bar{b}_{p}$ respectively in the first and second summand of the sum $\left[U^{h}(p t) / 2 U^{h}(p t)\right] \oplus$ $\left[U^{h}(p t) / 2 U^{h}(p t)\right]$. Since $A M(D)+B N(D)=0$ we have $\bar{a}_{p}=0, \bar{b}_{p}=0$ and thus $a_{p} \in 2 U^{*}(p t), b_{p} \in 2 U^{*}(p t)$.

(ii) $p<q$. From $J^{4 p+2,2 n-4 p-2} \supset J^{4 p+3,2 n-4 p-3} \supset J^{4 q+2,2 n-4 q-2}$ it follows that $g(B N(D))=0$ and consequently $\bar{a}_{p}=0$ which means that $a_{p} \in 2 U^{*}(p t)$.

(b) Suppose $E(D) \neq 0$.

Take $E(Z)=d_{0}+\sum_{i \geq 1} d_{i} Z^{i}$. As $E(D)=-(A M(D)+B N(D)) \in$ $\tilde{U}^{*}(B \Gamma)$ we have $d_{0}=0$. Hence:

$$
E(Z)=\sum_{i \geq r} d_{i} Z^{i}, \quad d_{r} \neq 0, \quad r \geq 1
$$

If $d_{r}=8 e_{r_{1}}$, we form

$$
\begin{aligned}
E_{1}(Z) & =E(Z)-e_{r_{1}} Z^{r-1} T(Z) \\
& =\sum_{i \geq r^{\prime}} d_{i}^{\prime} Z^{i}, \quad r^{\prime}>r, d_{r^{\prime}}^{\prime} \neq 0 \text { or } \nu\left(E_{1}\right)>\nu(E) .
\end{aligned}
$$

If $d_{r^{\prime}}^{\prime}=8 e_{r_{2}}$ we form $E_{2}(Z)=E_{1}(Z)-e_{r_{2}} Z^{r^{\prime}-1} T(Z)$ and so on. But after a finite number of steps we have $E_{p_{0}}(Z)=\sum_{i \geq h} t_{i} Z^{i}$ and $t_{h} \notin 8 U^{*}(p t)$ because, if not, we would have $E(Z) \in \Omega_{*} T(Z)$ and thus $E(D)=0$ which contradicts the hypothesis (b): $E(D) \neq 0$ (see the proof of 2.12). Hence there is a formal power series $F(Z) \in \Omega_{2 n}$ such that $F(D)=E(D)$ and $F(Z)=\sum_{i \geq h \geq 1} t_{i} Z^{i}, t_{h} \notin 8 U^{*}(p t)$. This means that $E(D) \in J^{4 h, 2 n-4 h}$ and $E(D) \notin J^{4 h+1,2 n-4 h-1}$. 
(i) $p=q, 4 h<4 p+2=4 q+2$.

Then: $J^{4 h, 2 n-4 h} \supset J^{4 h+1,2 n-4 h-1} \supset J^{4 p+2,2 n-4 p-2}$. Since $E(D)=$ $-(A M(D)+B N(D))$ we have $E(D) \in J^{4 h+1,2 n-4 h-1}$ which is impossible.

(ii) $p=q, 4 p+2=4 q+2<4 h$.

Then $J^{4 p+2,2 n-4 p-2} \supset J^{4 p+3,2 n-4 p-3} \supset J^{4 h, 2 n-4 h}$ and $A M(D)+$ $B N(D)=-E(D) \in J^{4 p+3,2 n-4 p-3}$. Consequently $\bar{a}_{p}=0, \bar{b}_{p}=0$ and thus $a_{p} \in 2 U^{*}(p t), b_{p} \in 2 U^{*}(p t)$.

(iii) $p<q, 4 h<4 p+2<4 q+2$.

Then $J^{4 h, 2 n-4 h} \supset J^{4 p+2,2 n-4 p-2} \supset J^{4 q+2,2 n-4 q-2}$. From $E(D)=$ $-(A M(D)+B N(D))$ it follows that

$$
E(D) \in J^{4 p+2,2 n-4 p-2} \subset J^{4 h+1,2 n-4 h-1}\left(\subset J^{4 h, 2 h-4 h}\right)
$$

which is impossible.

(iv) $p<q, 4 p+2<4 h<4 q+2$ or $4 p+2<4 q+2<4 h$.

We have either

$$
J^{4 p+2,2 n-4 p-2} \supset J^{4 p+3,2 n-4 p-3} \supset J^{4 h, 2 n-4 h} \supset J^{4 q+2,2 n-4 q-2}
$$

or

$$
J^{4 p+2,2 n-4 p-2} \supset J^{4 p+3,2 n-4 p-3} \supset J^{4 q+2,2 n-4 q-2} \supset J^{4 h, 2 n-4 h} .
$$

It follows in both cases that $\bar{a}_{p}=0$ and $a_{p} \in 2 U^{*}(p t)$. Hence we have proved that if $p \leq q$ we have $a_{p} \in 2 U^{*}(p t)$ in both cases $E(D)=0, E(D) \neq 0$. So $M(Z)=a_{p} Z^{p}+a_{p+1} Z^{p+1}+\cdots, a_{p}=2 e_{p} \neq 0$. By 2.13 if $K(X, Z)=X(2+J(Z))$ then $K(A, D)=0$. We form $X M(Z)-e_{p} Z^{p} K(X, Z)=X M_{1}(Z), M_{1}(Z)=e_{p_{1}} Z^{p_{1}}+\cdots, p_{1}>p$, and we get: $A M_{1}(D)+B N(D)+E(D)=0$. If $p_{1}<q$ we carry on the same process and after a finite number of steps there is $M_{r}(Z) \in \Lambda_{2 n-2}$ such that $A M_{r}(D)+B N(D)+E(D)=0$ and $q \leq p_{r}, p_{r}$ being such that $M_{r}(Z)=\omega_{p_{r}} Z^{p_{r}}+\omega_{p_{r}+1} Z^{p_{r}+1}+\cdots, \omega_{p_{r}} \neq 0$. Thus the argument used is the case $p \leq q$ (above) shows that $b_{q} \in 2 U^{*}(p t)$.

Let $I_{*}^{\prime}$ the graded ideal of $\Lambda_{*}$ generated by $K(X, Z)=X(2+J(Z)) \in$ $\Lambda_{2}, K(Y, Z)=Y \cdot(2+J(Z)) \in \Lambda_{2}$ and $T(Z) \in \Omega_{4}($ see $2.13,2.12)$.

LEMMA 2.15. Let $M(Z), N(Z), E(Z)$ be elements of $\Omega_{*}$ such that $A M(D)+B N(D)+E(D)=0$. Then: $X M(Z)+Y N(Z)+E(Z) \in$ $K(X, Z) \Omega_{*}+K(Y, Z) \Omega_{*}+T(Z) \Omega_{*} \subset I_{*}^{\prime}$ and $A M(D)=B N(D)=$ $E(D)=0$.

Proof. Suppose $X M(Z) \in \Lambda_{2 n}, Y N(Z) \in \Lambda_{2 n}, E(Z) \in \Lambda_{2 n}, n \in \mathbb{Z}$. We shall give a proof in the case $M(Z) \neq 0, N(Z) \neq 0$, the other cases 
being simpler. Take $P(X, Y, Z)=X M(Z)+Y N(Z)+E(Z), M(Z)=$ $a_{p_{0}} Z^{p_{0}}+a_{p_{0}+1} Z^{p_{0}+1}+\cdots, a_{p_{0}} \neq 0, N(Z)=b_{q_{0}} Z^{q_{0}}+b_{q_{0}} Z^{q_{0}+1}+\cdots$, $b_{q_{0}} \neq 0$. By 2.14 we have $a_{p_{0}}=2 a_{p_{0}}^{\prime}, b_{q_{0}}=2 b_{q_{0}}^{\prime}$ and then: $P(X, Y, Z)-$ $\left(a_{p_{0}}^{\prime} Z^{p_{0}} K(X, Z)+b_{q_{0}}^{\prime} Z^{q_{0}} K(Y, Z)\right)=X\left[M(Z)-a_{p_{0}}^{\prime} Z^{p_{0}}(2+J(Z))\right]+$ $Y\left[N(Z)-b_{q_{0}}^{\prime} Z^{q_{0}}(2+J(Z))\right]+E(Z)=X M_{1}(Z)+Y N_{1}(Z)+E(Z)$ with $\nu(M)<\nu\left(M_{1}\right), \nu(N)<\nu\left(N_{1}\right)$. Moreover we have $A M_{1}(D)+$ $B N_{1}(D)+E(D)=P(A, B, D)=0$. The same process can be carried out for $X M_{1}(Z)+Y N_{1}(Z)+E(Z)$ and after a finite number of operations we get $M_{1}(Z), M_{2}(Z), \ldots, M_{r+1}(Z), N_{1}(Z), N_{2}(Z), \ldots, N_{r+1}(Z)$,

$$
\begin{gathered}
P(X, Y, Z)-\left[\left(\sum_{i=0}^{r} a_{p_{i}}^{\prime} Z^{p_{i}}\right) K(X, Z)+\left(\sum_{i=0}^{r} b_{q_{i}}^{\prime} Z^{q_{i}}\right) K(Y, Z)\right] \\
=X M_{r+1}(Z)+Y N_{r+1}(Z)+E(Z)
\end{gathered}
$$

with $p_{0}=\nu^{\prime}(M)<p_{1}=\nu^{\prime}\left(M_{1}\right)<\cdots<p_{r+1}=\nu^{\prime}\left(M_{r+1}\right), q_{0}=$ $\nu^{\prime}(N)<q_{1}=\nu^{\prime}\left(N_{1}\right)<\cdots<q_{r+1}=\nu^{\prime}\left(N_{r+1}\right)$. Take

$$
H_{1}(Z)=\sum_{i=0}^{\infty} a_{p_{t}}^{\prime} Z^{p_{i}}, \quad H_{2}(Z)=\sum_{i=0}^{\infty} b_{q_{i}}^{\prime} Z^{q_{l}} .
$$

Since $\operatorname{Lim}_{r \rightarrow \infty} \nu\left(M_{r}\right)=\operatorname{Lim}_{r \rightarrow \infty} \nu\left(N_{r}\right)=+\infty$ we have $\operatorname{Lim}_{r \rightarrow \infty} X M_{r}(Z)$ $=\operatorname{Lim}_{r \rightarrow \infty} Y N_{r}(Z)=0$ and there are $H_{1}(Z) \in \Omega_{*}, H_{2}(Z) \in \Omega_{*}$ such that: $P(X, Y, Z)-\left[H_{1}(Z) K(X, Z)+H_{2}(Z) K(Y, Z)\right]=E(Z)$. Since $P(A, B, D)=K(A, D)=K(B, D)=0$ we have: $E(D)=0$ and then by 2.12 there is $H_{3}(Z) \in \Omega_{*}$ such that $E(Z)=H_{3}(Z) \cdot T(Z)$. Finally we have $P(X, Y, Z)=H_{1}(Z) K(X, Z)+H_{2}(Z) K(Y, Z)+H_{3}(Z) T(Z) \in$ $K(X, Z) \Omega_{*}+K(Y, Z) \Omega_{*}+T(Z) \Omega_{*} \subset I_{*}^{\prime}$ and $X M(Z)=H_{1}(Z) K(X, Z)$, $Y N(Z)=H_{2}(Z) \cdot K(Y, Z), E(Z)=H_{3}(Z) \cdot T(Z)$. Consequently: $A M(D)=B N(D)=E(D)=0$.

Consider $S(X, Z)=X^{2}-X S(Z) \in \Lambda_{4}, S(Y, Z)=Y^{2}-Y S(Z) \in \Lambda_{4}$, $R(X, Y, Z)=X Y-(X+Y)(P(Z)-S(Z))+Q(Z) \in \Lambda_{4}$. By 2.10 we have: $S(A, D)=S(B, D)=R(A, B, D)=0$. Let $I_{*}^{\prime \prime}$ be the grade ideal of $\Lambda_{*}$ generated by $S(X, Z), S(Y, Z), R(X, Y, Z)$.

LemmA 2.16. For any $P(X, Y, Z) \in \Lambda_{*}$ there are $M(Z), N(Z)$, $E(Z)$, elements of $\Omega_{*}$ such that $P(X, Y, Z)-[X M(Z)+Y N(Z)+$ $E(Z)] \in I_{*}^{\prime \prime}$.

Proof. From $X^{2}-X S(Z)=S(X, Z)$ we see that there is $M_{n}(X, Z) \in$ $\Lambda_{*}$ such that $X^{n}-X S^{n-1}(Z)=S(X, Z) M_{n}(X, Z), n \geq 2$, with $M_{2}(X, Z)$ $=1$ and $M_{n+1}(X, Z)=S^{n-1}(Z)+X M_{n}(X, Z), n \geq 2$. It is easily seen that $\operatorname{Lim}_{n \rightarrow \infty} \nu\left(S^{n}\right)=\operatorname{Lim}_{n \rightarrow \infty} \nu\left(M_{n}\right)=+\infty$. If $P(X, Y, Z) \in \Lambda_{2 m}$ we 
can write $P(X, Y, Z)=\sum_{i=0}^{\infty} X^{i} P_{i}(Y, Z)$ with $\operatorname{dim} P_{i}=2(m-i)$. We have $X^{i} P_{i}(Y, Z)=X S^{i-1}(Z) P_{i}(Y, Z)+S(X, Z) M_{i}(X, Z) P_{i}(Y, Z), i \geq$ 2. From Section $I$ and the fact that the multiplication by an element of $\Lambda_{*}$ is continuous we see that there are $H(Y, Z), H_{1}(X, Y, Z)$ such that: $P(X, Y, Z)=X H(Y, Z)+S(X, Z) H_{1}(X, Y, Z)+P_{0}(Y, Z)$. Similarly there are $F_{0}(Z), F_{1}(Z), F_{2}(Y, Z)$ such that $H(Y, Z)=Y F_{1}(Z)+$ $S(Y, Z) F_{2}(Y, Z)+F_{0}(Z)$ and $G_{0}(Z), G_{1}(Z), G_{2}(Y, Z)$ such that $P_{0}(Y, Z)=Y G_{1}(Z)+S(Y, Z) G_{2}(Y, Z)+G_{0}(Z)$. Then a straightforward calculation shows that with $M(Z)=F_{0}(Z)+F_{1}(Z) \cdot(P(Z)-$ $S(Z)), N(Z)=G_{1}(Z)+F_{1}(Z) \cdot(P(Z)-S(Z)), E(Z)=G_{0}(Z)-$ $Q(Z) \cdot F_{1}(Z)$ we get $P(X, Y, Z)-[X M(Z)+Y N(Z)+E(Z)] \in I_{*}^{\prime \prime}$.

Let $I_{*}$ be $I_{*}^{\prime}+I_{*}^{\prime \prime}$.

TheOREM 2.17. The graded $U^{*}(p t)$-algebra $U^{*}(B \Gamma)$ is isomorphic to $\Lambda_{*} / I_{*}$ where $I_{*}$ is a graded ideal generated by six homogeneous formal power series.

Proof. Consider the map $\varphi: \Lambda_{*} \rightarrow U^{*}(B \Gamma)$ of graded $U^{*}(p t)$-algebras such that $\varphi(X)=A, \varphi(Y)=B, \varphi(Z)=D$. By Theorem $2.5 \varphi$ is surjective and by Lemmas 2.15, $2.16 \varphi$ is injective.

REMARKs. (1) Consider the involution $h: \Lambda_{*} \rightarrow \Lambda_{*}$ such that $h(Y)=$ $X, h(X)=Y, H(Z)=Z$. We have $h\left(I_{*}\right)=I_{*}$ and thus there is an isomorphism $\bar{h}$ of graded $U^{*}(p t)$-algebras: $U^{*}(B \Gamma) \rightarrow U^{*}(B \Gamma)$ such $\bar{h}(A)=B, \bar{h}(B)=A, \bar{h}(D)=D$. Consequently $\bar{h}^{2}=$ Id.

(2) If $q: \mathbb{Z}_{2} \subset \Gamma$ denotes the canonical inclusion, then $(B q)^{*}: U^{*}(B \Gamma)$ $\rightarrow U^{*}\left(B \mathbb{Z}_{2}\right)$ is neither injective nor surjective.

An important and easy consequences of Theorem 2.12 and Lemma 2.15 is the following theorem which gives the structure of $U^{*}(p t)[[D]]-$ module of $U^{*}(B \Gamma)$.

THEOREM 2.18. (a) As graded $U^{*}(p t)$-algebras we have:

$$
U^{*}(p t)[[D]] \simeq \Omega_{*} /(T(Z)) .
$$

(b) As graded $U^{*}(p t)[[D]]-$ modules we have: $U^{*}(B \Gamma) \simeq U^{*}(p t)[[D]]$ $\oplus U^{*}(p t)[[D]] A \oplus U^{*}(p t)[[D]] . B$ and: $A$ and $B$ have the same annihilator

$$
(2+J(D)) U^{*}(p t)[[D]] .
$$

III. Computation of $U^{*}\left(B \Gamma_{k}\right), k \geq 4$. The group $\Gamma_{k}, k \geq 4$, is generated by $u, v$, subject to the following relations $u^{t}=v^{2}, u v u=v$, 
$t=2^{k-2} ;\left|\Gamma_{k}\right|=2^{k}$. We have $H^{0}\left(B \Gamma_{k}\right)=\mathbb{Z}, H^{4 p}\left(B \Gamma_{k}\right)=\mathbb{Z}_{2^{k}}$, $p>0, H^{4 p+2}=\mathbb{Z}_{2} \oplus \mathbb{Z}_{2}, p \geq 0, H^{2 p+1}\left(B \Gamma_{k}\right)=0, p \geq 0$. Furthermore if $d_{1},\left\{a_{1}, b_{1}\right\}$ are generators of respectively $H^{4}\left(B \Gamma_{k}\right)$ and $H^{2}\left(B \Gamma_{k}\right)$, then $d_{1}^{p},\left\{a_{1} d_{1}^{p}, b_{1} d_{1}^{p}\right\}$ are generators of respectively $H^{4 p}\left(B \Gamma_{k}\right)$ and $H^{4 p+2}\left(B \Gamma_{k}\right), p \geq 0$ (see [5]). The irreducible unitary representations of $\Gamma_{k}$ are $1: u \rightarrow 1, v \rightarrow 1, \xi_{1}: u \rightarrow 1, v \rightarrow-1, \xi_{2}: u \rightarrow-1, v \rightarrow 1$, $\xi_{3}: u \rightarrow-1, v \rightarrow-1$,

$$
\eta_{r}: u \rightarrow\left(\begin{array}{cc}
\omega^{r} & 0 \\
0 & \omega^{-r}
\end{array}\right), \quad v \rightarrow\left(\begin{array}{cc}
0 & (-1) \\
1 & 0
\end{array}\right), \quad r=1,2, \ldots, 2^{k-2}-1
$$

and $\omega$ a primitive $2^{k-1}$ th root of unity (see [6]).

The relations between the irreducible unitary representations of $\Gamma_{k}$ are as follows: $\xi_{1}^{2}=\xi_{2}^{2}=\xi_{3}^{2}=1, \xi_{1} \cdot \xi_{2}=\xi_{3}, \xi_{2} \xi_{3}=\xi_{1}, \xi_{3} \cdot \xi_{1}=$ $\xi_{2}$; if we introduce $\eta_{0}=1+\xi_{1}, \eta_{2^{k-2}}=\xi_{2}+\xi_{3}$, then we can define $\eta_{s}, s \in \mathbb{Z}$, by the relations $\eta_{2^{k-2}+r}=\eta_{2^{k-2}-r}, \eta_{r}=\eta_{-r}$ and we have: $\eta_{r} \cdot \eta_{s}=\eta_{r+s}+\eta_{r-s}, r \in \mathbb{Z}, s \in \mathbb{Z}$ (see [10]). As in Section II we shall be working with $A_{k}=c f_{1}\left(\xi_{1}\right) \in \tilde{U}^{2}\left(B \Gamma_{k}\right), B_{k}=c f_{1}\left(\xi_{2}\right) \in \tilde{U}^{2}\left(B \Gamma_{k}\right)$, $C_{k}=c f_{1}\left(\xi_{3}\right) \in \tilde{U}^{2}\left(B \Gamma_{k}\right), D_{k}=c f_{2}\left(\eta_{1}\right) \in \tilde{U}^{4}\left(B \Gamma_{k}\right)$. We have as in 2.5 with $U^{*}(p t)\left[\left[D_{k}\right]\right]=\left\{R\left(D_{k}\right), R \in \Omega_{*}\right\}$ :

TheOREM 3.1. $U^{*}\left(B \Gamma_{k}\right)$ is concentrated in even dimensions and as a module over $U^{*}(p t)\left[\left[D_{k}\right]\right], U^{*}\left(B \Gamma_{k}\right)$ is generated by $1, B_{k}, C_{k}$.

The following proposition is proved in the same way as 2.8 and 2.6, $P_{0}(Z)$ being the formal power series of 2.8:

Proposition 3.2. (a) We have $c f_{1}\left(\eta_{1}\right)=P_{0}\left(D_{k}\right)$.

(b) If $H(Z)=\sum_{i \geq 0} \alpha_{i} Z^{i} \in \Omega_{2 n}$ is such that $H\left(D_{k}\right)=0$, then $\alpha_{0}=0$ and the leading coefficient of $H(Z)$ belongs to $2^{k} U^{*}(p t)$.

LEMMA 3.3. For each $n \in \mathbb{Z}$ there is a polynomial $P_{2 n+1}(X) \in \mathbb{Z}[X]$ such that $P_{2 n+1}(0)=0, P_{2 n+1}(2)=2, P_{2 n+1}\left(\eta_{1}\right)=\eta_{2 n+1}$.

Proof. Since $\eta_{-r}=\eta_{r}$, we may suppose $n \geq 0$. Then the assertion is evidently true if $n=0$ with $P_{1}(X)=X$. Suppose that there are polynomials $P_{2 i+1}(X) \in \mathbb{Z}[X], 0 \leq i \leq n-1$, such that $P_{2 i+1}\left(\eta_{1}\right)=\eta_{2 i+1}, P_{2 i+1}(0)=0$ and $P_{2 i+1}(2)=2$. Then $\eta_{1}^{2} P_{2 n-1}\left(\eta_{1}\right)=$ $\eta_{1}^{2} \eta_{2 n-1}=\left(\eta_{2}+\eta_{0}\right) \eta_{2 n-1}=\eta_{2 n+1}+2 \eta_{2 n-1}+\eta_{2 n-3}$. Hence if $P_{2 n+1}(X)=$ $\left(X^{2}-2\right) P_{2 n-1}(X)-P_{2 n-3}(X)$ we have $P_{2 n+1}(X) \in \mathbb{Z}[X], P_{2 n+1}(0)=0$, $P_{2 n+1}(2)=2$ and $P_{2 n+1}\left(\eta_{1}\right)=\eta_{2 n+1}$. 
In the sequel we shall consider the sequence $P_{2 n+1}, n \geq 0$, determined by $P_{1}(X)=X, P_{3}(X)=X^{3}-3 X$ and the relation

$$
\left(X^{2}-2\right) P_{2 n-1}(X)-P_{2 n-3}(X)=P_{2 n+1}(X) .
$$

If $P(X) \in \mathbb{Z}[X]$ we shall denote by $P^{\prime}$ the derivatives of $P$.

Proposition 3.4. If $\zeta$ is a complex vector bundle over $B \Gamma_{k}$ such that $\zeta=P\left(\eta_{1}\right)$ where $P \in \mathbb{Z}[X], P(0)=0$, then there is a formal power series $P^{\prime}(2) Z+\sum_{i \geq 2} \delta_{i} Z^{i} \in \Omega_{4}$ such that $c f_{2}(\zeta)=P^{\prime}(2) D_{k}+\sum_{i \geq 2} \delta_{i} D_{k}^{i}$.

Proof. For each $q \geq 1$ the complex bundle $\eta_{1}^{q}$ is classified by the composite: $\Gamma_{k} \stackrel{\Delta}{\rightarrow}\left(B \Gamma_{k}\right)^{q} \stackrel{X^{q} g}{\rightarrow}(B U(2))^{q} \stackrel{m_{q}}{\rightarrow} B U\left(2^{q}\right)$ where $\Delta$ is the diagonal map, $g$ a map classifying $\eta_{1}$ and $m_{q}$ a map classifying $\otimes^{q} \gamma(2)$. We have $U^{*}\left(B U(2)^{q}\right)=U^{*}(p t)\left[\left[c_{1}^{(1)}, c_{2}^{(1)}, c_{1}^{(2)}, c_{2}^{(2)}, \ldots, c_{1}^{(q)}, c_{2}^{(q)}\right]\right]$ where $c_{k}^{(i)}, k=1$ or $k=2$, is the image of $a_{1} \otimes a_{2} \cdots \otimes a_{q}, a_{1}=a_{2}=$ $\cdots=a_{i-1}=1, a_{i}=c f_{k}(\gamma(2))(k=1$ or $k=2), a_{i+1}=\cdots=a_{q}=$ 1 , by the canonical product $\otimes^{q} U^{*}(B U(2)) \rightarrow U^{*}\left(B U\left(2^{q}\right)\right)$. Then $m_{q}^{*}\left(c f_{2} \gamma\left(2^{q}\right)\right)=\sum a_{u}\left(c_{1}^{(1)}\right)^{u_{1}^{(1)}} \cdot\left(c_{2}^{(1)}\right)^{u_{2}^{(1)}} \cdots\left(c_{1}^{(q)}\right)^{u_{1}^{(q)}} \cdot\left(c_{2}^{(q)}\right)^{u_{2}^{(q)}}$. If we substitute $Z$ for $c_{2}^{(i)}$ and $P_{0}(Z)$ for $c_{1}^{(l)}, i=1,2, \ldots, q$, we have a formal power series $R_{q}(Z) \in \Omega_{4}$ such that $R_{q}\left(D_{k}\right)=c f_{2}\left(\eta_{1}^{q}\right)$. If $\left\{p_{j}\right\}$ denotes the base point of $B U(2)$ and $k_{i}$ the inclusion:

$\left\{p_{1}\right\} \times\left\{p_{2}\right\} \times \cdots \times\left\{p_{i-1}\right\} \times B U(2) \times\left\{p_{i+1}\right\} \times \cdots \times\left\{p_{q}\right\} \subset(B U(2))^{q}$,

we see that $k_{i}^{*} \circ m_{q}^{*}\left(c f_{2}\left(\gamma\left(2^{q}\right)\right)\right)=c f_{2}\left(2^{q-1} \gamma(2)\right)=2^{q-1} c f_{2}(\gamma(2))+$ $2^{q-2}\left(2^{q-1}-1\right) c f_{1}^{2}(\gamma(2))$. Consequently $R_{q}(Z)=q 2^{q-1} Z+\sum_{i \geq 2} \varepsilon_{i} Z^{i}$. Similarly there are formal powers series $H_{1}(Z) \in \Omega_{2}, H_{s}(Z) \in \Omega_{2 s}, s \geq$ 3, such that $H_{1}\left(D_{k}\right)=c f_{1}\left(\eta_{1}^{q}\right)$ and $H_{s}\left(D_{k}\right)=c f_{s}\left(\eta_{1}^{q}\right), s \geq 3$; we have $\nu^{\prime}\left(H_{1}\right) \geq 1, \nu^{\prime}\left(H_{S}\right) \geq 2, s \geq 3$. (We recall that $\nu^{\prime}(P(Z))=\frac{1}{4} \nu P(Z)$.) It follows that if $\zeta=\sum_{i=1}^{r} m_{i} \eta_{1}^{i}, m_{i} \geq 0$, there is a formal power series $H(Z) \in \Omega_{4}$ such that $H\left(D_{k}\right)=c f_{2}(\zeta)$ and $H(Z)=\left(\sum_{i=1}^{r} i m_{i} 2^{i-1}\right) Z+$ $\sum_{i \geq 2} \varepsilon_{i}^{\prime} Z^{i}$. Now suppose that $\zeta$ is a complex vector bundle such that $\zeta=\sum_{i=1}^{r} m_{i} \eta_{1}^{i}-\sum_{i=1} n_{i} \eta_{1}^{i}, m_{i} \geq 0, n_{i} \geq 0$. The above remarks show that

$$
\begin{aligned}
c f(\zeta)= & 1+c f_{1}(\zeta)+c f_{2}(\zeta)+\cdots \\
= & {\left[1+M_{1}\left(D_{k}\right)+c f_{2}\left(\zeta_{1}\right)+M_{2}\left(D_{k}\right)\right] } \\
& \times\left[1+M_{1}^{\prime}\left(D_{k}\right)+c f_{2}\left(\zeta_{2}\right)+M_{2}^{\prime}\left(D_{k}\right)\right]^{-1}
\end{aligned}
$$

with $\zeta_{1}=\sum_{i=1}^{r} m_{i} \eta_{1}^{i}, \zeta_{2}=\sum_{i=1}^{r} n_{i} \eta_{1}^{i}, M_{1}, M_{2}, M_{1}^{\prime}, M_{2}^{\prime}$ being elements of $\Omega_{*}$ such that $\nu^{\prime}\left(M_{1}\right) \geq 1, \nu^{\prime}\left(M_{1}^{\prime}\right) \geq 1, \nu^{\prime}\left(M_{2}\right) \geq 2, \nu^{\prime}\left(M_{2}^{\prime}\right) \geq$ 2. It follows that $c f_{2}(\zeta)=M\left(D_{k}\right)$, with $M(Z) \in \Omega_{4}$ and $M(Z)=$ 
$\sum_{i=1}^{r}\left(i m_{i} 2^{i-1}-i n_{i} 2^{i-1}\right) Z+\sum_{i \geq 2} \delta_{i} Z^{i}$. Then if $P(X)=\sum_{i=1}^{r} m_{i} X^{i}-$ $\sum_{i=1}^{r} n_{i} X^{i} \in \mathbb{Z}[X]$ we see that $M(Z)=P^{\prime}(2) Z+\sum_{i \geq 2} \delta_{i} Z^{i}, P^{\prime}(X)$ being the derivative of $P(X)$.

LEMMA 3.5. There is a formal power series

$$
Q_{1}(Z)=\left(1+2^{2} n(n+1)\right) Z+\sum_{i \geq 2} \beta_{i}^{\prime} Z^{i} \in \Omega_{4}
$$

such that $Q_{1}\left(D_{k}\right)=c f_{2}\left(\eta_{2 n+1}\right)$.

Proof. Since $\eta_{2 n+1}=P_{2 n+1}\left(\eta_{1}\right)$ with $P_{2 n+1} \in \mathbb{Z}[X], P_{2 n+1}(0)=0$, then by 3.4 it is enough to prove that $P_{2 n+1}^{\prime}(2)=1+2^{2} n(n+1)$. This assertion is true when $n=0$ because $P_{1}(X)=X$. Suppose that $P_{2 i+1}^{\prime}(2)=1+2^{2} i(i+1), 0 \leq i \leq n-1$. We have $P_{2 n+1}=\left(X^{2}-2\right) P_{2 n-1}-$ $P_{2 n-3}$ and then $P_{2 n+1}^{\prime}(2)=2^{2} P_{2 n-1}(2)+2 P_{2 n-1}^{\prime}(2)-P_{2 n-3}^{\prime}(2)=2^{3}+$ $2\left[1+2^{2}(n-1) n\right]-\left[1+2^{2}(n-2)(n-1)\right]=1+2^{2} n(n+1)\left(P_{2 n-1}(2)=2\right.$ by 3.3$)$. Hence the lemma has been proved.

In Lemma 3.5 the coefficients $\beta_{i}^{\prime}$ depend on $n$; however we have chosen not to complicate the notation.

Proposition 3.6. There is a formal power series

$$
\begin{aligned}
T_{k}(Z)= & 2^{k} Z+2^{k-2} \lambda_{2}^{\prime} Z^{2}+2^{k-3} \lambda_{3}^{\prime} Z^{3} \\
& +\cdots+2 \lambda_{k-1}^{\prime} Z^{k-1}+\sum_{i \geq k} \lambda_{i}^{\prime} Z^{i} \in \Omega_{4},
\end{aligned}
$$

with $\lambda_{2}^{\prime} \notin 2 U^{*}(p t)$, such that $T_{k}\left(D_{k}\right)=0$. Moreover if $R(Z) \in \Omega_{*}$ and $R\left(D_{k}\right)=0$ then $R(Z) \in T_{k}(Z) \Omega_{*}$.

Proof. From 3.5 there is a formal power series

$$
Q_{1}(Z)=\left[1+2^{2}\left(2^{k-3}-2\right)\left(2^{k-3}-1\right)\right] Z+\sum_{i \geq 2} \beta_{i}^{\prime} Z^{i} \in \Omega_{4}
$$

such that $Q_{1}\left(D_{k}\right)=c f_{2}\left(\eta_{2^{k-2}-3}\right)$. We have $1+2^{2}\left(2^{k-3}-2\right)\left(2^{k-3}-1\right)=$ $9+2^{2 k-4}-3 \cdot 2^{k-1}$. Now

$$
\begin{aligned}
\eta_{1}^{2} \eta_{2^{k-2}-1} & =\left(\eta_{2}+\eta_{0}\right) \eta_{2^{k-2}-1} \\
& =\eta_{2^{k-2}+1}+\eta_{2^{k-2}-3}+2 \eta_{2^{k-2}-1}=3 \eta_{2^{k-2}-1}+\eta_{2^{k-2}-3}
\end{aligned}
$$

and consequently if $P(X)=\left(X^{2}-3\right) P_{2^{k-2}-1}$, we have $P \in \mathbb{Z}[X], P(0)=$ 0 and $P\left(\eta_{1}\right)=\eta_{2^{k-2}-3}$. Then from 3.4 there is a formal power series $Q_{2}(Z)=P^{\prime}(2)+\sum_{i \geq 2} \beta_{i}^{\prime \prime} Z^{i} \in \Omega_{4}$ such that $Q_{2}\left(D_{k}\right)=c f_{2}\left(\eta_{2^{k-2}-3}\right)$. We 
have $P^{\prime}(2)=2^{2} P_{2^{k-2}-1}(2)+P_{2^{k-2}-1}^{\prime}(2)=2^{3}+1+2^{2}\left(2^{k-3}-1\right) 2^{k-3}=$ $9+2^{2 k-4}-2^{k-1}$. Hence

$$
\begin{aligned}
0= & Q_{2}\left(D_{k}\right)-Q_{1}\left(D_{k}\right) \\
= & {\left[9+2^{2 k-4}-2^{k-1}-\left(9+2^{2 k-4}-3 \cdot 2^{k-1}\right)\right] D_{k} } \\
& +\sum_{i \geq 2}\left(\beta_{i}^{\prime \prime}-\beta_{i}^{\prime}\right) D_{k}^{i} \\
= & 2^{k} D_{k}+\sum_{i \geq 2} \mu_{i}^{\prime} D_{k}^{i}, \quad \mu_{i}^{\prime}=\beta_{i}^{\prime \prime}-\beta^{\prime} .
\end{aligned}
$$

Then if $T_{k}(Z)=2^{k} Z+\sum_{i \geq 2} \mu_{i}^{\prime} Z^{i} \in \Omega_{4}$ then we have $0=T_{k}\left(D_{k}\right)$. By 3.2 and a proof similar to that of 2.12, Section II, if $R(Z) \in \Omega_{*}$ is such that $R\left(D_{k}\right)=0$ then $R(Z) \in T_{k}(Z) \Omega_{*}$. Now we want to show that $\mu_{2}^{\prime}=2^{k-2} \lambda_{2}^{\prime}, \lambda_{2}^{\prime} \notin 2 U^{*}(p t), \mu_{3}^{\prime}=2^{k-3} \lambda_{3}^{\prime}, \ldots, \mu_{k-1}^{\prime}=2 \lambda_{k-1}^{\prime}$. Instead of $T_{3}(Z)$ we take the formal power series $T(Z)$ defined in Section II (see 2.11). We recall that $T(Z)=2^{3} Z+2 \lambda_{2} Z^{2}+\sum_{i \geq 3} \lambda_{i} Z^{i}, \lambda_{2} \notin 2 U^{*}(p t)$. Hence if $k=3$ the assertion concerning the coefficients of $T_{k}(Z)$ is true. Suppose that

$$
\begin{aligned}
T_{k}(Z)= & 2^{k} Z+2^{k-2} \lambda_{2}^{\prime} Z^{2}+2^{k-3} \lambda_{3}^{\prime} Z^{3} \\
& +\cdots+2 \lambda_{k-1}^{\prime} Z^{k-1}+\sum_{i \geq k} \lambda_{i}^{\prime} Z^{i}, \quad \lambda_{2}^{\prime} \notin 2 U^{*}(p t) .
\end{aligned}
$$

Consider the inclusion

$$
\begin{aligned}
i_{k+1}: \Gamma_{k} & =\left\{\left(u^{2}\right)^{m} v^{n}, n=0,1,0 \leq m \leq 2^{k-1}-1\right\} \subset \Gamma_{k+1} \\
& =\left\{u^{m} v^{n}, n=0,1,0 \leq m \leq 2^{k}-1\right\} .
\end{aligned}
$$

It is easily seen that $\left(B i_{k+1}\right)^{*}\left(D_{k+1}\right)=D_{k}$. We have: $T_{k+1}(Z)=$ $2^{k+1} Z+\sum_{i \geq 2} \mu_{i}^{\prime \prime} Z^{i}$ and $T_{k+1}\left(D_{k+1}\right)=0$. It follows that $T_{k+1}\left(D_{k}\right)=0$ and consequently there is an element $\alpha_{0}^{\prime}+\alpha_{1}^{\prime} Z+\alpha_{2}^{\prime} Z^{2}+\cdots \in \Omega_{0}$ such that:

$$
\begin{aligned}
& 2^{k+1} Z+\sum_{i \geq 2} \mu_{i}^{\prime \prime} Z^{i} \\
& \quad=\left(2^{k} Z+2^{k-2} \lambda_{2}^{\prime} Z^{2}+\cdots+2 \lambda_{k-1}^{\prime} Z^{k-1}+\sum_{i \geq k} \lambda_{i}^{\prime} Z^{i}\right)\left(\sum_{i \geq 0} \alpha_{i}^{\prime} Z^{i}\right) .
\end{aligned}
$$

Then $\alpha_{0}^{\prime}=2 ; \mu_{2}^{\prime \prime}=2^{k} \alpha_{1}^{\prime}+2^{k-2} \lambda_{2}^{\prime} \alpha_{0}^{\prime}=2^{k-1}\left[2 \alpha_{1}^{\prime}+\lambda_{2}^{\prime}\right]=2^{k-1} \lambda_{2}^{\prime \prime}, \lambda_{2}^{\prime \prime} \notin$ $2 U^{*}(p t)$; if $2 \leq i \leq k$ we have:

$\mu_{i}^{\prime \prime}=2^{k} \alpha_{i-1}^{\prime}+2^{k-2} \lambda_{2}^{\prime} \alpha_{i-2}^{\prime}+2^{k-3} \lambda_{3}^{\prime} \alpha_{i-3}^{\prime}+\cdots+2^{k-i} \lambda_{i}^{\prime} \alpha_{0}^{\prime}=2^{(k+1)-i} \lambda_{i}^{\prime \prime}$.

Hence the proposition has been proved. 
Suppose $k \geq 4$; the inclusions $i_{k}: \Gamma_{k-1} \subset \Gamma_{k}$ and $j_{k}: \Gamma \subset \Gamma_{k}$ are given respectively by $\left\{\left(u^{2}\right)^{m} v^{n}, 0 \leq m \leq 2^{k-2}-1, n=0,1\right\} \subset$ $\left\{u^{m} v^{n}, 0 \leq m \leq 2^{k-1}-1, n=0,1\right\}$ and $j_{k}=i_{k} \circ \cdots \circ i_{4} ; \Gamma_{k}$ is normal in $\Gamma_{k+1}$ and $\Gamma_{k+1} / \Gamma_{k}=\{1, \bar{u}\} \simeq \mathbb{Z}_{2}$; if $q_{k}: \Gamma_{k} \rightarrow \Gamma_{k}$ is the conjugation by $u \in \Gamma_{k+1}-\Gamma_{k}$ then $q_{k}\left(u^{2}\right)=u^{2}, q_{k}(v)=v\left(u^{2}\right)^{-1}$. Let $f_{k}: B \Gamma_{k} \rightarrow B \Gamma_{k-1}, g_{k}: B \Gamma \rightarrow B \Gamma_{k}, h_{k}: B \Gamma_{k} \rightarrow B \Gamma_{k}$ be respectively $B i_{k}, B j_{k}$ and $B q_{k}$.

LEMMA 3.7. Suppose $k \geq 4$.

(a) $f_{k}^{*}\left(A_{k}\right)=A_{k-1}, f_{k}^{*}\left(B_{k}\right)=0, f_{k}^{*}\left(C_{k}\right)=A_{k-1}, f_{k}^{*}\left(D_{k}\right)=D_{k-1}$.

(b) $g_{k}^{*}\left(A_{k}\right)=A, g_{k}^{*}\left(B_{k}\right)=0, g_{k}^{*}\left(C_{k}\right)=A, g_{k}^{*}\left(D_{k}\right)=D$.

(c) $h_{k}^{*}\left(A_{k}\right)=A_{k}, h_{k}^{*}\left(B_{k}\right)=C_{k}, h_{k}^{*}\left(C_{k}\right)=B_{k}$.

Proof. The proof is easy; for example $f_{k}^{*}\left(A_{k}\right)=A_{k-1}$ because $i_{k}^{*}$ : $R\left(\Gamma_{k}\right) \rightarrow R\left(\Gamma_{k-1}\right)$ maps $\xi_{1}$ to the similar representation: $u^{2} \rightarrow 1$, $v \rightarrow-1$. $\left(R\left(\Gamma_{k}\right)\right.$ and $R\left(\Gamma_{k-1}\right)$ denote the representation rings).

The role played by $A, B, C$ in Section II was symmetrical. Unfortunately this is not the case for $A_{k}, B_{k}, C_{k}(k \geq 4)$ as we shall see in the forthcoming propositions. We recall that there are formal power series $S(Z) \in \Omega_{2}, J(Z) \in \Omega_{0}$ such that $A^{2}=A S(D), B^{2}=B S(D)$, $C^{2}=C S(D), A(2+J(D))=B(2+J(D))=C(2+J(D))=0$ (see 2.10, 2.13).

The formal power series $S(Z), J(Z)$ will play an important role in the calculations ahead.

PROPOSITION 3.8. Suppose $k \geq 4$.

(a) $A_{k} B_{k} C_{k}=0$.

(b) $A_{k}\left(2+J\left(D_{k}\right)\right)=0$.

(c) There are $E_{k} \in \Omega_{2}, F_{k} \in \Omega_{4}$ such that $A_{k}=B_{k}+C_{k}-E_{k}\left(D_{k}\right)$, $B_{k} C_{k}=F_{k}\left(D_{k}\right)$.

Proof. (a) The relation $A_{k} B_{k} C_{k}=0$ is proved in exactly the same way as in $2.9(\mathrm{~b})$.

(b) By 3.1 there are $H_{0}(Z) \in \Omega_{2}, H_{1}(Z) \in \Omega_{2}, H_{2}(Z) \in \Omega_{4}$ such that: $B_{k+1}^{2}=B_{k+1} H_{0}\left(D_{k+1}\right)+C_{k+1}\left(D_{k+1}\right)+H_{2}\left(D_{k+1}\right)$. By $3.7(\mathrm{c})$ we get $C_{k+1}^{2}=C_{k+1} H_{0}\left(D_{k+1}\right)+B_{k+1} H_{1}\left(D_{k+1}\right)+H_{2}\left(D_{k+1}\right)$ and $C_{k+1}^{2}-B_{k+1}^{2}=$ $\left(C_{k+1}-B_{k+1}\right) H_{3}\left(D_{k+1}\right)$ with $H_{3}=H_{0}-H_{1} \in \Omega_{2}$. By using 3.7(a) we see that: $A_{k}^{2}=A_{k} \cdot H_{3}\left(D_{k}\right)$; as in 2.13 the relation $c f_{1}\left(\xi_{1}^{2}\right)=$ 0 shows that there is $J_{1}(Z) \in \Omega_{0}$ depending on $H_{3}(Z)$ such that $A_{k}\left(2+J_{1}\left(D_{k}\right)\right)=0$ and by $3.7(\mathrm{~b})$ we get $A\left(2+J_{1}(D)\right)=0$; so there is 
$H_{4}(Z) \in \Omega_{0}, \nu^{\prime}\left(H_{4}\right) \geq 1$ such that $2+J_{1}(Z)=(2+J(Z))\left(1+H_{4}(Z)\right)$ (see 2.15) and consequently $2+J(Z)=\left(2+J_{1}(Z)\right) H_{5}(Z)$, $H_{5}(Z) \in \Omega_{0}$ being such that: $\left(1+H_{4}(Z)\right)\left(1+H_{5}(Z)\right)=1$. Hence $A_{k}\left(2+J\left(D_{k}\right)\right)=0$.

(c) By using the relations $\eta_{r} \cdot \eta_{s}=\eta_{r+s}+\eta_{r-s}, r \in \mathbb{Z}, s \in \mathbb{Z}, \eta_{0}=1+\xi_{1}$, $\eta_{2^{k-2}}=\xi_{2}+\xi_{3}$, then a straightforward calculation shows that there is a polynomial $R_{m}[X] \in \mathbb{Z}[X]$ such that $R_{m}(0)=0$ and $\eta_{2^{m}}=R_{m}\left(\eta_{1}\right)+\eta_{0}$, $2 \leq m \leq k-2$; in fact $R_{m}(X)$ is determined by $R_{2}(X)=X^{4}-4 X$, $R_{m}(X)=R_{m-1}^{2}(X)+4 R_{m-1}(X)$; so: $\xi_{2}+\xi_{3}=\eta_{2^{k-2}}=R_{k-2}\left(\eta_{1}\right)+\eta_{0}=$ $R_{k-2}\left(\eta_{1}\right)+1+\xi_{1}$. Then the proof of 3.4 shows that there are $E_{k}(Z) \in$ $\Omega_{2}, F_{k}(Z) \in \Omega_{4}$ such that: $B_{k}+C_{k}=c f_{1}\left(R_{k-2}\left(\eta_{1}\right)\right)+A_{k}=E_{k}\left(D_{k}\right)+$ $A_{k}$ and $B_{k} C_{k}=A_{k} E_{k}\left(D_{k}\right)+c f_{2}\left(R_{k-2}\left(\eta_{1}\right)\right)=A_{k} E_{k}\left(D_{k}\right)+F_{k}\left(D_{k}\right)$. As $0=A E_{k}(D)+F_{k}(D)$ by $3.7(\mathrm{~b})$ we see that $E_{k}(Z) \in(2+J(Z)) \Omega_{*}$ and consequently $B_{k} C_{k}=F_{k}(D)$ since $A_{k}\left(2+J\left(D_{k}\right)\right)=0$. Hence (c) is proved.

Proposition 3.9. Suppose $k \geq 4$.

(a) There is $M(Z) \in \Omega_{2}$ such that: $B_{k}\left(2+J\left(D_{k}\right)\right)+M\left(D_{k}\right)=$ $C_{k}\left(2+J\left(D_{k}\right)\right)+M\left(D_{k}\right)=0$ and $M\left(D_{k}\right) \neq 0$.

(b) There is $N(Z) \in \Omega_{4}$, such that: $B_{k}^{2}=B_{k} S\left(D_{k}\right)+N\left(D_{k}\right), C_{k}^{2}=$ $C_{k} S\left(D_{k}\right)+N\left(D_{k}\right)$ and $N\left(D_{k}\right) \neq 0$.

(c) There are $G_{k}(Z) \in \Omega_{2}, L_{k}(Z) \in \Omega_{4}$ the coefficients of which can be computed from those of $J(Z), S(Z), E_{k}(Z), F_{k}(Z)$ and satisfying $G_{k}\left(D_{k}\right)=M\left(D_{k}\right), L_{k}\left(D_{k}\right)=N\left(D_{k}\right)$.

Proof. (a) As in 3.1 there are $H_{1}(Z) \in \Omega_{2}, K_{0}(Z) \in \Omega_{2}, K_{1}(Z) \in \Omega_{4}$ such that: $B_{k}^{2}=B_{k} H_{1}\left(D_{k}\right)+A_{k} K_{0}\left(D_{k}\right)+K_{1}\left(D_{k}\right)$; hence: $A K_{0}(D)=0$ which imply by 2.15 that $K_{0}(Z) \in(2+J(Z)) \Omega_{*}$; so: $B_{k}^{2}=B_{k} H_{1}\left(D_{k}\right)+$ $K_{1}\left(D_{k}\right)$ because $A_{k}\left(2+J\left(D_{k}\right)\right)=0$ by $3.8(\mathrm{~b})$. We have $B_{k}^{n+1}=$ $B_{k} H_{n}\left(D_{k}\right)+K_{n}\left(D_{k}\right)$ with $H_{n}(Z) \in \Omega_{2 n}, K_{n}(Z) \in \Omega_{2 n+2}$ satisfying: $H_{n}(Z)=H_{1}(Z) H_{n-1}(Z)+K_{n-1}(Z), K_{n}(Z)=K_{1}(Z) H_{n-1}(Z), n \geq$ 2. It follows easily that $\operatorname{Lim}_{n \rightarrow \infty} \nu\left(H_{n}\right)=\operatorname{Lim}_{n \rightarrow \infty} \nu\left(K_{n}\right)=+\infty$; as $c f_{1}\left(\xi_{2}^{2}\right)=0$ we have $2 B_{k}+\sum_{n \geq 2} a_{n} B_{k}^{n}=0$ with $a_{n}=\sum_{i+j=n} a_{i j}$, the $a_{i j}, i \geq 1, j \geq 1$, being the coefficients of the formal group law. A proof similar to that of 2.13 shows that there are $P_{1}(Z) \in \Omega_{0}, P_{2}(Z) \in \Omega_{2}$, $\nu^{\prime}\left(P_{1}\right) \geq 1, \nu^{\prime}\left(P_{2}\right) \geq 1$ such that $B_{k}\left(2+P_{1}\left(D_{k}\right)\right)+P_{2}\left(D_{k}\right)=0$; by 3.7(a) we have $C_{k}\left(2+P_{1}\left(D_{k}\right)\right)+P_{2}\left(D_{k}\right)=0$; hence $A\left(2+P_{1}(D)\right)=0$ and as a direct consequence of 2.15 there is $P_{3}(Z) \in \Omega_{0}$ such that $2+J(Z)=\left(2+P_{1}(Z)\right) P_{3}(Z)$ and then: $B_{k}\left(2+J\left(D_{k}\right)\right)+M\left(D_{k}\right)=$ $C_{k}\left(2+J\left(D_{k}\right)\right)+M\left(D_{k}\right)=0$ with $M(Z)=P_{2}(Z) . P_{3}(Z) \in \Omega_{2}$. Suppose $M\left(D_{k}\right)=0$; then $B_{k}\left(2+J\left(D_{k}\right)\right)=C_{k}\left(2+J\left(D_{k}\right)\right)=0$; from 
3.8(c) we have $A_{k}^{2}=A_{k}\left(B_{k}+C_{k}\right)-A_{k} E_{k}\left(D_{k}\right)$ and consequently $A E_{k}(D)=0$; so $E_{k}(Z) \in(2+J(Z)) \Omega_{*}$ and $A_{k}^{2}=\left(B_{k}+C_{k}\right)^{2}$. Let $\theta: M U \rightarrow K$ being the canonical map between spectra; $\theta$ sends Euler classes to Euler classes; the relation $A_{k}^{2}=\left(B_{k}+C_{k}\right)^{2}$ becomes by using $\theta: 1+\xi_{1}-\xi_{2}-\xi_{3}=0$ in $K^{0}\left(B \Gamma_{k}\right)$ which is impossible since $1+\xi_{1}-\xi_{2}-\xi_{3} \neq 0$ in $R\left(\Gamma_{k}\right)$ (the canonical map from $R\left(\Gamma_{k}\right)$ to $K^{0}\left(B \Gamma_{k}\right)$ is injective). Hence $M\left(D_{k}\right) \neq 0$.

(b) We have seen in (a) that $B_{k}^{2}=B_{k} H_{1}\left(D_{k}\right)+K_{1}\left(D_{k}\right)$; so: $C_{k}^{2}=$ $C_{k} H_{1}\left(D_{k}\right)+K_{1}\left(D_{k}\right)$ and: $A^{2}=A H_{1}(D)+K_{1}(D)=A S(D)$; then $A\left[H_{1}(D)-S(D)\right]+K_{1}(D)=0$ and there is $S_{0}(Z) \in \Omega_{2}$ such that $H_{1}(Z)=S(Z)+(2+J(Z)) S_{0}(Z)$; consequently: $B_{k}^{2}=B_{k} S\left(D_{k}\right)-$ $M\left(D_{k}\right) S_{0}\left(D_{k}\right)+K_{1}\left(D_{k}\right)=B_{k} S\left(D_{k}\right)+N\left(D_{k}\right)$ with: $N(Z)=K_{1}(Z)-$ $M(Z) S_{0}(Z) \in \Omega_{4}$; by 3.7(c) $C_{k}^{2}=C_{k} S\left(D_{k}\right)+N\left(D_{k}\right)$. If $N\left(D_{k}\right)=0$ then as in 2.13 we would have $C_{k}\left(2+J\left(D_{k}\right)\right)=0$ and then $M\left(D_{k}\right)=0$ which is false by (a). Hence: $N\left(D_{k}\right) \neq 0$.

(c) We need to show first that $T_{k}(Z) \notin 2 \Omega_{*}\left(T_{3}(Z)=T(Z)\right.$ and $T_{k}(Z)$ are defined respectively in 2.11 and 3.6). Suppose $k=3$; from $A B+B C+C A=Q(D)$ and $A(2+J(D))=B(2+J(D))=0$ (see 2.9 and 2.13) we get $(2+J(D)) Q(D)=0$; so:

$$
\begin{aligned}
(2+J(Z)) Q(Z)= & \left(2+\mu_{1} Z+\mu_{2} Z^{2}+\cdots\right)\left(4 Z+\beta_{2} Z^{2}+\beta_{3} Z^{3}+\cdots\right) \\
= & 8 Z+\left(2 \beta_{2}+4 \mu\right) Z^{2}+\left(2 \beta_{3}+\mu_{1} \beta_{2}+4 \mu_{2}\right) Z^{3} \\
& +\cdots \in T(Z) \Omega_{*} ;
\end{aligned}
$$

hence $T(Z) \notin 2 \Omega_{*}$ since $\mu_{1} \beta_{2} \notin 2 U^{*}(p t)$ (see 2.9 and 2.13). Suppose that $T_{i}(Z) \notin 2 \Omega_{*}, 3 \leq i \leq k-1$, and $T_{k}(Z) \in 2 \Omega_{*}$; as $A_{k}=$ $B_{k}+C_{k}-E_{k}\left(D_{k}\right)\left(\right.$ see 3.8(c)) we have $E_{k}\left(D_{k-1}\right)=0$ and then $E_{k}(Z) \in$ $T_{k-1}(Z) \Omega_{*}$; from $T_{k}(Z) \in T_{k-1}(Z) \Omega_{*}, T_{k}(Z) \in 2 \Omega_{*}$ and $T_{k-1}(Z) \notin$ $2 \Omega_{*}$ it follows easily that $2 T_{k-1}\left(D_{k}\right)=0$; consequently $2 E_{k}\left(D_{k}\right)=0$ and $2 A_{k}=2\left(B_{k}+C_{k}\right)$ which is impossible (it can be seen by using $\theta: M U \rightarrow K$ as in (a)). Hence $T_{k}(Z) \notin 2 \Omega_{*}, k \geq 3$. Let $q: \Omega_{*} \rightarrow \Omega_{*} / 2 \Omega_{*}=\left(U^{*}(p t) / 2 U^{*}(p t)\right)[[Z]]$ be the canonical projection and $\bar{R}(Z)$ the image of $R(Z)$ by $q$. Now it follows easily from 3.8(c) and (a) that: $2 M\left(D_{k}\right)+E_{k}\left(D_{k}\right)\left(2+J\left(D_{k}\right)\right)=0$ and then $2 M(Z)+E_{k}(Z)(2+J(Z))=T_{k}(Z) \cdot H(Z), H(Z) \in \Omega_{*}$. Hence $\bar{E}_{k}(Z) \cdot \bar{J}(Z)=\bar{T}_{k}(Z) \cdot \bar{H}(Z)$; as $\bar{T}_{k}(Z) \neq 0$ the formal power series $\bar{H}(Z)$ is unique and its coefficients which belong to $U^{*}(p t) / 2 U^{*}(p t)=$ $\mathbb{Z}_{2}\left[x_{1}, x_{1}, \ldots\right]\left(\left|x_{i}\right|=-2 i\right)$ are computable from those of $\bar{E}_{k}, \bar{J}$ and $\bar{T}_{k}$; if $\bar{H}(Z)=\sum \bar{d}_{i} Z^{i}, \bar{d}_{i} \neq 0$, then there is a unique element $e_{i} \in \mathbb{Z}\left[x_{1}, \ldots, x_{n}, \ldots\right]=U^{*}(p t)$ whose coefficients as a polynomial in $x_{1}, \ldots, x_{n}, \ldots$, are odd and such that $\bar{e}_{i}=\bar{d}_{i}$; it follows that 
$E_{k}(Z)(2+J(Z))-T_{k}(Z) \cdot\left(\sum e_{i} Z^{i}\right)=-2 G_{k}(Z)$ and $G_{k}\left(D_{k}\right)=M\left(D_{k}\right)$. The same method can be used to determine $L_{k}(Z)$ by considering the relation $2 N\left(D_{k}\right)=E_{k}^{2}\left(D_{k}\right)-E_{k}\left(D_{k}\right) S\left(D_{k}\right)-2 F\left(D_{k}\right)$ which is an easy consequence of $(\mathrm{b})$ and 3.8(c).

Let $\tilde{I}_{*}^{\prime}$ be the graded ideal of $\Lambda_{*}$ generated by the homogeneous formal power series $G_{k}(X, Z)=X(2+J(Z))+G_{k}(Z) \in \Lambda_{2}, G_{k}(Y, Z)=$ $Y(2+J(Z))+G_{k}(Z) \in \Lambda_{2}, T_{k}(Z) \in \Lambda_{4}$ (see 3.6 and 3.9) and $\tilde{I}_{*}^{\prime \prime}$ the graded ideal of $\Lambda_{*}$ generated by the homogeneous formal power series $L_{k}(X, Z)=X^{2}-X S(Z)-L_{k}(Z) \in \Lambda_{4}, L_{k}(Y, Z)=Y^{2}-Y S(Z)-$ $L_{k}(Z) \in \Lambda_{4}, X Y-F_{k}(Z) \in \Lambda_{2}$ (see 3.8(c) and 3.9). The proofs of the following lemmas are quite similar to those of $2.15,2.16$ and will be omitted.

LEMMA 3.10. If $H_{1}(Z), H_{2}(Z), H_{3}(Z)$ are elements of $\Omega_{*}$ such that $B_{k} H_{1}\left(D_{k}\right)+C_{k} H_{2}\left(D_{k}\right)+H_{3}\left(D_{k}\right)=0$ then $X H_{1}(Z)+Y H_{2}(Z)+H_{3}(Z) \in$ $G_{k}(X, Z) \Omega_{*}+G_{k}(Y, Z) \Omega_{*}+T_{k}(Z) \Omega_{*} \subset \tilde{I}_{*}^{\prime}$.

LeMmA 3.11. For any $P(X, Y, Z) \in \Lambda_{*}$ there are $H_{1}(Z), H_{2}(Z)$, $H_{3}(Z)$ elements of $\Omega_{*}$ such that $P(X, Y, Z)-\left[X H_{1}(Z)+Y H_{2}(Z)+\right.$ $\left.H_{3}(Z)\right] \in \tilde{I}_{*}^{\prime \prime}$.

As a direct consequence of $3.10,3.11$ we get our main theorem where $\tilde{I}_{*}=\tilde{I}_{*}^{\prime}+\tilde{I}_{*}^{\prime \prime}$ (see the proof of 2.17 ).

THEOREM 3.12. The graded $U^{*}(p t)$-algebra $U^{*}\left(B \Gamma_{k}\right)$ is isomorphic to $\Lambda_{*} / \tilde{I}_{*}$ where $\tilde{I}_{*}$ is a graded ideal of $\Lambda_{*}$ generated by six homogeneous formal power series.

REMARK. The homomorphism $f_{k}^{*}$ induced by the inclusion $\Gamma_{k-1} \subset$ $\Gamma_{k}$ (see 3.7) is such that $f_{k}^{*}\left(B_{k}\right)=0$,

$$
f_{k}^{*}\left(C_{k}\right)=B_{k-1}+C_{k-1}-E_{k-1}\left(D_{k-1}\right)\left(E_{k-1}\left(D_{k-1}\right) \neq 0\right),
$$

$f_{k}^{*}\left(D_{k}\right)=D_{k-1}$ if $k \geq 5$ (see 3.8). But $f_{4}^{*}\left(B_{4}\right)=0, f_{4}^{*}\left(C_{4}\right)=P(D)-$ $(B+C), P(D) \neq 0$ (see 2.9, 2.6), $f_{4}^{*}\left(D_{4}\right)=D$.

Let $U^{*}(p t)\left[\left[D_{k}\right]\right]$ be $\left\{R\left(D_{k}\right), R(Z) \in \Omega_{*}\right\}$.

THEOREM 3.13. (a) $U^{*}(p t)\left[\left[D_{k}\right]\right] \simeq \Omega_{*} /\left(T_{k}\right)$ as graded $U^{*}(p t)$ algebras.

(b) $U^{*}\left(B \Gamma_{k}\right)$ is generated by $1, A_{k}, B_{k}$ as a $U^{*}(p t)\left[\left[D_{k}\right]\right]-$ module. Moreover if $V_{k}=U^{*}(p t)\left[\left[D_{k}\right]\right]$ then:

$$
V_{k} \cap V_{k} B_{k}=V_{k} \cap V_{k} C_{k}=V_{k} B_{k} \cap V_{k} C_{k}=G_{k}\left(D_{k}\right) \cdot V_{k} .
$$


Proof. The assertion (a) is a consequence of 3.6; the first part of (b) is proven in 3.1 and the second part is a consequence of 3.10.

Now we are going to alter $B_{k}, C_{k}$ in order to improve $3.13(\mathrm{~b})$. From $B_{k}\left(2+J\left(D_{k}\right)\right)+G_{k}\left(D_{k}\right)=0$ it follows easily that $G_{k}(D)=0$; so $A G_{k}(D)=0$ and $G_{k}(Z)=(2+J(Z)) G_{k}^{\prime}(Z), G_{k}^{\prime}(Z) \in \Omega_{2}$; hence

$$
\left(B_{k}+G_{k}^{\prime}\left(D_{k}\right)\right)\left(2+J\left(D_{k}\right)\right)=\left(C_{k}+G_{k}^{\prime}\left(D_{k}\right)\right)\left(2+J\left(D_{k}\right)\right)=0 .
$$

Furthermore if $\mu: U^{*}\left(B \Gamma_{k}\right) \rightarrow H^{*}\left(B \Gamma_{k}\right)$ is the edge homomorphism (in connection with the $U^{*}$-AHSS for $\left.B \Gamma_{k}\right)$ then $\mu\left(B_{k}+G_{k}^{\prime}\left(D_{k}\right)\right)=$ $\mu\left(B_{k}\right), \mu\left(C_{k}+G_{k}^{\prime}\left(D_{k}\right)\right)=\mu\left(C_{k}\right)$. This remark and Lemma 3.10 allow the following rearrangement of Theorem 3.13 with $B_{k}^{\prime}=B_{k}+G_{k}^{\prime}\left(D_{k}\right)$, $C_{k}^{\prime}=C_{k}+G_{k}^{\prime}\left(D_{k}\right)$.

TheOREM 3.14. (a) $U^{*}(p t)\left[\left[D_{k}\right]\right] \simeq \Omega_{*} /\left(T_{k}\right)$ as graded $U^{*}(p t)$ algebras.

(b) As graded $U^{*}(p t)\left[\left[D_{k}\right]\right]$-modules we have:

$$
U^{*}\left(B \Gamma_{k}\right) \simeq U^{*}(p t)\left[\left[D_{k}\right]\right] \oplus U^{*}(p t)\left[\left[D_{k}\right]\right] \cdot B_{k}^{\prime} \oplus U^{*}(p t)\left[\left[D_{k}\right]\right] \cdot C_{k}^{\prime}
$$

and $B_{k}^{\prime}, C_{k}^{\prime}$ have the same annihilator $\left(2+J\left(D_{k}\right)\right) \cdot U^{*}(p t)\left[\left[D_{k}\right]\right]$.

\section{Appendix.}

(A) Calculation of $U^{*}\left(B \mathbb{Z}_{m}\right)$ by a new method. The method used in the case $G=\Gamma_{k}$ applies more simply in the case $G=\mathbb{Z}_{m}$. Let $w$ be $\exp (2 i / m)$ and $\rho$ the irreducible unitary representation of $\mathbb{Z}_{m}$ defined by $\rho(\bar{q})=w^{q}, \bar{q} \in \mathbb{Z}_{m}$. Let $\eta$ be the complex vector bundle over $B \mathbb{Z}_{m}$ corresponding to $\rho$ and $D_{1}=e(\eta)=c f_{1}(\eta) \in U^{2}\left(B \mathbb{Z}_{m}\right)$.

Let $\Lambda_{*}^{\prime}$ be $U^{*}(p t)[[Z]]$, graded by taking $\operatorname{dim} Z=2$. There is a topology on $\Lambda_{2 n}^{\prime}, n \geq 0$, defined by the subgroups $J_{r}=\left\{P \in \Lambda_{2 n}^{\prime}, \nu(P) \geq\right.$ $r\}$, with $\nu(P)=2 s$ if $P(Z)=a_{s} Z^{s}+a_{s+1} Z^{s+1}+\cdots, a_{s} \neq 0 ; \Lambda_{2 n}^{\prime}$ is complete and Hausdorff. Furthermore, $U^{2 n}\left(B \mathbb{Z}_{m}\right)$ is topologized by the subgroups $J^{r, 2 n-r}$ induced by the $U^{*}$-AHSS for $B \mathbb{Z}_{m}$, taken as a system of neighbourhoods of 0 . The group $U^{2 n}\left(B \mathbb{Z}_{m}\right)$ is complete and Hausdorff because the $U^{*}$-AHSS for $B \mathbb{Z}_{m}$ collapses. Moreover there is a unique continuous homomorphism of graded $U^{*}(p t)$ algebras $\varphi^{\prime}: \Lambda_{*}^{\prime} \rightarrow U^{*}\left(B \mathbb{Z}_{m}\right)$ such that $\varphi^{\prime}(Z)=D_{1}$ and $\varphi^{\prime}$ is surjective (see Sections I and II).

The complex vector bundle $\eta^{m}$ is trivial $\left(\operatorname{dim} \eta^{m}=1\right)$ because $\rho^{m}=$ 1. Hence $c f_{1}\left(\eta^{m}\right)=0$. If $m_{0}$ denotes a map: $B U(1)^{m} \rightarrow B U(1)$ classifying $\otimes^{m} \gamma(1)(\gamma(1)$ being a universal complex vector bundle over $B U(1))$ and if $c_{1}=c f_{1}(\gamma(1))$ then:

$$
m_{0}^{*}\left(c_{1}\right)=\sum a_{(u)} e_{1}^{u_{1}} e_{2}^{u_{2}} \cdots e^{u_{m}}, \quad u=\left(u_{1}, \ldots, u_{m}\right) .
$$


$u_{1} \geq 0, \ldots, u_{m} \geq 0, e_{i}$ being the image of $a_{1} \otimes a_{2} \otimes \cdots \otimes a_{m}$ with $a_{1}=a_{2}=\cdots a_{i-1}=1, a_{i}=c_{1}, a_{i+1}=\cdots=a_{m}=1$, by the product: $\left.\otimes^{m} U^{*}(B U(1)) \rightarrow U B U(1)^{m}\right)$. The vector bundle $\eta^{m}$ is classified by the composite:

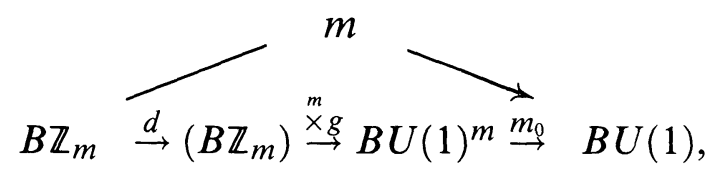

$d$ being the diagonal map and $g$ a map classifying $\eta$. It follows that if $T(Z)=\sum a_{(u)} Z^{u_{1}+u_{2}+\cdots u_{m}} \in \Lambda_{2}^{\prime}$, we have $T\left(c f_{1}(\eta)\right)=T(e(\eta))=$ $T\left(D_{1}\right)=0$. It is easily seen that $T(Z)=[\mathrm{m}](Z)$.

THEOREM A.1. $U^{*}\left(B \mathbb{Z}_{m}\right) \simeq \Lambda_{*}^{\prime} /([m](Z))$ as graded $U^{*}(p t)$-algebras.

Proof. Let $I_{*}$ be $([m](Z))$. The homomorphism $\varphi^{\prime}: \Lambda_{*}^{\prime} \rightarrow U^{*}\left(B \mathbb{Z}_{m}\right)$ of graded $U^{*}(p t)$-algebras, defined above, is surjective; moreover $\varphi^{\prime}\left(I_{*}\right)$ $=0$. Hence $\varphi^{\prime}$ gives rise to a homomorphism of graded $U^{*}(p t)$ algebras $\bar{\varphi}^{\prime}: \Lambda_{*}^{\prime} / I_{*} \rightarrow U^{*}\left(B \mathbb{Z}_{m}\right)$. Let $P(Z)$ be any element of $\Lambda_{2 n}^{\prime}$ $(n \geq 0)$ such that $P\left(D_{1}\right)=0$; if $P(Z)=a_{0}+a_{1} Z+a_{2} Z^{2}+\cdots$, then $a_{0}=0$ because $a_{0}=-\left(a_{1} D_{1}+a_{2} D_{1}^{2}+\cdots\right) \in \tilde{U}^{*}\left(B \mathbb{Z}_{m}\right) \cap U^{*}(p t)=0$. It follows that $P(Z)=a_{p_{0}} Z^{p_{0}}+a_{p_{0}+1} Z^{p_{0}+1}+\cdots$, with $p_{0} \geq 1, a_{p_{0}} \neq 0$. We have

$$
a_{p_{0}+1} D_{1}^{p_{0}+1}+\cdots+a_{p_{0}+k} D_{1}^{p_{0}+k} \in J^{2\left(p_{0}+1\right), 2\left(n-p_{0}-1\right)}
$$

since this group is closed in $U^{2 n}\left(B \mathbb{Z}_{m}\right)$, it follows that

$$
\sum_{i=1}^{\infty} a_{p+i} D_{1}^{p_{0}+i} \in J^{2\left(p_{0}+1\right), 2\left(n-p_{0}-1\right)} \subset J^{2 p_{0}+1,2\left(n-p_{0}\right)-1} .
$$

Let $s$ be the quotient map:

$$
\begin{aligned}
& J^{2 p_{0}, 2\left(n-p_{0}\right)} \rightarrow J^{2 p_{0}, 2\left(n-p_{0}\right)} / J^{2 p_{0}+1,2\left(n-p_{0}\right)-1} \\
& \quad=H^{2 p_{0}}\left(B \mathbb{Z}_{m}\right) \otimes U^{2\left(n-p_{0}\right)}(p t)=\mathbb{Z}_{m} \otimes U^{2\left(n-p_{0}\right)}(p t) \\
& \quad=U^{2\left(n-p_{0}\right)}(p t) / m U^{2\left(n-p_{0}\right)}(p t)
\end{aligned}
$$

$\left(H^{2 p_{0}}\left(B \mathbb{Z}_{m}\right)=\mathbb{Z}_{m}\right.$ because $\left.p_{0} \geq 1\right)$. It follows from $s\left(P\left(D_{1}\right)\right)=0$ that $a_{p_{0}}=m a_{p_{0}}^{\prime}$. We form $P_{1}(Z)=P(Z)-a_{p_{0}}^{\prime} Z^{p_{0}-1} T(Z)$; then $P_{1}\left(D_{1}\right)=0$ and $\nu\left(P_{1}\right)>\nu(P)$. We repeat the same process, and there is an element $P_{r+1}(Z) \in \Lambda_{2 n}^{\prime}, r \geq 1$, such that

$$
P_{r+1}(Z)=P(Z)-\left(a_{p_{0}}^{\prime} Z^{p_{0}-1}+a_{p_{1}}^{\prime} Z^{p_{1}-1}+\cdots+a_{p_{r}}^{\prime} Z^{p_{r}-1}\right) T(Z)
$$

with the properties: $P_{r+1}\left(D_{1}\right)=0, \nu\left(P_{r+1}\right)=p_{r+1}>p_{r} \cdots>p_{1}>$ $p_{0}$. Hence $\lim _{r \rightarrow \infty} \nu\left(P_{r+1}\right)=+\infty$ and by Sec. I we have $P(Z)=$ 
$\left(\sum_{i=0}^{\infty} a_{p_{i}}^{\prime} Z^{p_{t}-1}\right) T(Z) \in I_{2 n}$. It follows that $\bar{\varphi}^{\prime}$ is injective and the theorem has been proved.

Note. P. S. Landweber has proved a similar result by using other methods (see [13]).

(B) Calculation of $U^{*}(B S U(n))$. Particular case $n=2: S U(2)=$ $S p(1)$. Consider the $S^{1}$-bundle $U(n) / S U(n)=S^{1} \rightarrow B S U(n) \stackrel{p}{\rightarrow}$ $B U(n), n \geq 2, p=B i$ with $i: S U(n) \subset U(n)$; let $\xi$ be the complex vector bundle $E=B S U(n) \times_{S^{1}} \mathbb{C} \stackrel{\pi}{\rightarrow} B U(n)$, where $S^{1}$ acts on $\mathbb{C}$ by the multiplication in $\mathbb{C}$. If $E_{0}=E-j(B U(n)), j$ being the zero-section of $\xi$, then we have the Gysin exact sequence (see [4]):

$$
\begin{aligned}
\cdots & \rightarrow U^{i}(B U(n)) \stackrel{e(\xi)}{\rightarrow} U^{i+2}(B U(n)) \stackrel{\pi_{0}^{*}}{\rightarrow} U^{i+2}\left(E_{0}\right) \\
& \rightarrow U^{i+1}(B U(n)) \rightarrow \cdots,
\end{aligned}
$$

where $\pi_{0}$ denotes $\pi \mid E_{0}$. The map $g: B S U(n) \rightarrow E_{0}$ defined by $g(x)=$ $[x, 1]$ is an embedding; take $E^{\prime}=g(B S U(n)), j^{\prime}$ the inclusion: $E^{\prime} \subset$ $E_{0}$ and $h: E_{0} \rightarrow E^{\prime}$ the map defined by $h[x, z]=[x z /|z|, 1]$; then by using $h$ and the homotopy $H: E_{0} \times I \rightarrow E_{0}$ given by $H([x, z], t)=$ $[x, t z+(1-t) z /|z|]$ we see that $E^{\prime}$ is a strong deformation retract of $E_{0}$; it is easily seen that $\pi^{\prime} \circ h=\pi_{0}$ and $\pi^{\prime} \circ g=p$ with $\pi^{\prime}=\pi \mid E^{\prime}, g$ being considered as a homeomorphism: $B S U(n) \stackrel{\sim}{\rightarrow} g(B S U(n))$. So: $\pi_{0}^{*}=h^{*} \circ g^{*-1} \circ p^{*}$ and since $h^{*} \circ g^{*-1}$ is an isomorphism the above exact sequence gives the following one:

$$
\begin{aligned}
\cdots & \rightarrow U^{i}(B U(n)) \stackrel{\cdot(\xi)}{\rightarrow} U^{i+2}(B U(n)) \stackrel{p^{*}}{\rightarrow} U^{i+2}(B S U(n)) \\
& \rightarrow U^{i+1}(B U(n)) \rightarrow \cdots .
\end{aligned}
$$

Consider the canonical map of ring spectra $f: M U \rightarrow H$ (see [1]); $f^{\#}(-)$ maps Euler classes to Euler classes. Suppose $e(\xi)=0$; then $f^{\#}(-)(e(\xi))=0$, which means that the Euler class of $\xi$ for $H$ is 0 . From the Gysin exact sequence of $\xi$ for $H$ it follows easily that $H^{2}(B U(n)) \simeq H^{2}(B S U(n))$ which is impossible since $H^{2}(B U(n)) \neq 0$ and $H^{2}(B S U(n))=0$ (see [12], page 237). Hence $e(\xi) \neq 0$ and the map $\cdot-e(\xi)$ is injective. Consequently the sequence:

$$
0 \rightarrow U^{2 i}(B U(n)) \stackrel{\cdot e(\xi)}{\rightarrow} U^{2 i+2}(B U(n)) \stackrel{p^{*}}{\rightarrow} U^{2 i+2}(B S U(n)) \rightarrow 0
$$

is exact and $U^{2 i+1}(B S U(n))=0, i \geq 0$. So we have:

TheOREM B.1. We have $U^{2 i+1}(B S U(n))=0, i \geq 0$, and the map $p^{*}$ induces an isomorphism:

$$
U^{2 i+2}(B U(n)) / e(\xi) U^{2 i}(B U(n)) \simeq U^{2 i+2}(B S U(n)), \quad i \in \mathbb{Z} .
$$


Now let $\left(g_{i j}\right)$ be a set of transition functions for a universal $U(n)$ bundle: $E U(n) \rightarrow B U(n)$. If $\bar{g}_{i j}$ denotes the image of $g_{i j}$ by the quotient map $q: U(n) \rightarrow U(n) / S U(n)=S^{1}$ then $\left(\bar{g}_{i j}\right)$ is a set of transition functions for $\xi$; from $q\left(g_{i j}\right)=\operatorname{det}\left(g_{i j}\right)$ and $\operatorname{dim} \xi=1$, it follows that $\xi$ is isomorphic to the complex vector bundle $\Lambda^{n} \gamma(n), \gamma(n)$ being a universal vector bundle over $B U(n)$. Hence:

THEOREM B.2.

$$
U^{2 i+2}(B U(n)) / e\left(\Lambda^{n} \gamma(n)\right) \cdot U^{2 i}(B U(n)) \simeq U^{2 i+2}(B S U(n)) .
$$

and $U^{2 i+1}(B S U(n))=0, i \geq 0$.

Particular Case $n=2 ; S p(1)=S U(2)$. By Section II we have $U^{*}(B S p(1))=U^{*}(B S U(2))=U^{*}(p t)[[V]]$, with $V=c f_{2}(\theta), \theta$ being a universal $S p(1)$-vector bundle over $B S(1)$, regarded as a $U(2)$-vector bundle. Then $c f_{1}(\theta)=P_{0}(V)=\sum_{i=1}^{\infty} b_{i} V^{i} \in U^{2}(B S U(2))$. If $p$ denotes the projection: $B S U(2) \rightarrow B U(2)$, we have seen that the following sequence is exact: $0 \rightarrow U^{2 i}(B U(2)) \stackrel{\cdot e\left(\Lambda^{2} \gamma(2)\right)}{\rightarrow} U^{2 i+2}(B U(2)) \stackrel{p^{*}}{\rightarrow}$ $U^{2 i+2}(B S U(2)) \rightarrow 0$. We wish to calculate the coefficients $b_{i}, i \geq 1$. The $S p(1)$-vector bundle $\theta$ considered as a $S U(2)$-vector-bundle is a universal $S U(2)$-vector-bundle over $B S U(2)$ isomorphic to $p^{*}(\gamma(2))$ as a complex vector bundle. We have $U^{*}(B U(2))=U^{*}(p t)\left[\left[c_{1}, c_{2}\right]\right]$. $c_{1}=c f_{1}(\gamma(2)), c_{2}=c f_{2}(\gamma(2))$ and consequently

$$
\begin{aligned}
p^{*}\left(c_{1}\right) & =\sum_{i \geq 1} b_{i} V^{i}=\sum_{i \geq 1} b_{i}\left(c f_{2}(\theta)\right)^{i}=\sum_{i \geq 1} b_{i} p^{*}\left(c_{2}\right)^{i} \\
& =p^{*}\left(\sum_{i \geq 1} b_{i} c_{2}^{i}\right) .
\end{aligned}
$$

It follows that: $c_{1}-\sum_{i \geq 1} b_{i} c_{2}^{i}=e\left(\Lambda^{2} \gamma(2)\right) \cdot H\left(c_{1}, c_{2}\right)$ with $H\left(c_{1} c_{2}\right) \in$ $U^{0}(B U(2))$.

Let $k: B U(1) \times B U(1) \rightarrow B U(2)$ be a map classifying $\gamma(1) \times \gamma(1)$. Hence $k^{*}\left(\Lambda^{2} \gamma(2)\right)=\gamma(1) \otimes \gamma(1)$ and $k^{*}\left(e\left(\Lambda^{2} \gamma(2)\right)\right)=F(X, Y)$, the formal group law. Then $k^{*}\left(c_{1}-\sum_{i \geq 1} b_{i} c_{2}^{i}\right)=F(X, Y) k^{*}\left(H\left(c_{1}, c_{2}\right)\right)$; as $k^{*}\left(c_{1}\right)=X+Y$ and $k^{*}\left(c_{2}\right)=X Y$ we get:

$$
X+Y-\sum_{i \geq 1} b_{i}(X Y)^{i}=F(X, Y) G(X, Y) \in U^{*}(p t)[[X, Y]] .
$$

If $i(X)=[-1](X)$ then we have:

$$
X+i(X)=\sum_{i \geq 1} b_{i}(X \cdot i(X))^{i}
$$


This relation determines completely the coefficients $b_{i}, i \geq 1$; for example $b_{1}=-a_{11}, b_{2}=a_{11} a_{11} a_{21}-a_{22} \cdots$ the $a_{i j}$ being the coefficients of the group law.

(C) Ring Structure of $H^{*}\left(B \Gamma_{k}\right), k \geq 3$. M. Atiyah has determined the ring-structure of $H^{*}\left(B \Gamma_{3}\right)$ by using $K$-theory (see [2]); namely $H^{*}\left(B \Gamma_{3}\right)=\mathbb{Z}[x, y, z]$ subject to the relations $x y=4 z, 2 x=2 y=$ $x^{2}=y^{2}=8 z=0, \operatorname{dim} x=2, \operatorname{dim} y=2, \operatorname{dim} z=4$. We want to give another proof of this result using complex cobordism and determine the ring structure of $H^{*}\left(B \Gamma_{k}\right), k \geq 4$.

We have $H^{2}(B \Gamma)=\mathbb{Z} x \oplus \mathbb{Z} y, H^{4}(B \Gamma)=\mathbb{Z} \cdot z$ with $x=c_{1}\left(\xi_{j}\right)$, $y=c_{1}\left(\xi_{k}\right), z=c_{2}(\eta)$ (see Section II). Moreover: $2 x=2 y=8 z=0$. We have

$$
\begin{gathered}
B^{2}=B S(D), \quad C^{2}=C S(D), \\
B C=(B+C)[P(D)-S(D)]-Q(D)
\end{gathered}
$$

( $A, B, C$ play a symmetrical role; see Section II). If $\mu$ is the edge homomorphism we have $x^{2}=\mu(B S(D))=0\left(\mu: J^{4,0} \rightarrow J^{4,0} / J^{5,-1}=\right.$ $\left.H^{4}\left(B \Gamma_{3}\right) ; B S(D) \in J^{6,-2} \subset J^{5,-1}\right)$; similarly $y^{2}=0 ; x y=-\mu(Q(D))$ $=-4 z_{3}=-4 z=4 z$ because $Q(D)=4 D+\sum_{i \geq 2} \beta_{i} Z^{i}$ (see 2.9).

Suppose $k \geq 4$. We have $H^{2}\left(B \Gamma_{k}\right)=\mathbb{Z} x_{k} \oplus \mathbb{Z} y_{k}, H^{4}\left(B \Gamma_{k}\right)=$ $\mathbb{Z} \cdot z_{k}$ with $x_{k}=c_{1}\left(\xi_{2}\right), y_{k}=c_{1}\left(\xi_{3}\right), z_{k}=c_{2}\left(\eta_{1}\right)$ (see $\left.2.3,2.4\right)$. We have $2 x_{k}=2 y_{k}=2^{k} z_{k}=0$. The proof of Proposition 3.8 shows that $x_{k} y_{k}=\mu\left(F_{k}\left(D_{k}\right)\right), \mu$ being the edge homomorphism, $F_{k}\left(D_{k}\right)=$ $c f_{2}\left(R_{k-2}\left(\eta_{1}\right)\right)$ with $R_{k-2}(X) \in \mathbb{Z}[X] ; R_{k-2}(X)$ is determined inductively by $R_{2}(X)=X^{4}-4 X^{2}, R_{m+1}(X)=R_{m}^{2}(X)+4 R_{m}(X), m \geq 2$. By 3.4 we get $F_{k}\left(D_{k}\right)=R_{k-2}^{\prime}(2)+\sum_{i \geq 2} \nu_{i} D_{k}^{i}, \nu_{i} \in U^{*}(p t), R_{k-2}^{\prime}(X)$ being the derivative of $R_{k-2}(X)$. An easy calculation shows that $R_{k-2}^{\prime}(2)=$ $2^{2 k-4}$. As $2 k-4 \geq k$ we get $x_{k} y_{k}=2^{2 k-4} z_{k}=0$. As a consequence of the relations in $R\left(\Gamma_{k}\right)$ stated in the beginning of Section III we get: $\xi_{2} \eta_{1}=\eta_{2^{k-2}-1}$. Hence $x_{k}^{2}+c_{2}\left(\eta_{1}\right)=c_{2}\left(\eta_{2^{k-2}-1}\right)$ because $c_{1}\left(\eta_{1}\right)=0$. By $3.5 c f_{2}\left(\eta_{2^{k-2}-1}\right)=\left[1+2^{k-1}\left(2^{k-3}-1\right)\right] D_{k}+\sum_{i \geq 2} \beta_{i}^{\prime} D_{k}^{i}$ and consequently $c_{2}\left(\eta_{2^{k-2}-1}\right)=\left(1-2^{k-1}\right) z_{k}$. Therefore: $x_{k}^{2}=-2^{k-1} z_{k}=2^{k-1} z_{k}$. Similarly: $y_{k}^{2}=2^{k-1} z_{k}$. Hence we have proved the following result:

THEOREM C. If $k \geq 4$ we have $H^{*}\left(B \Gamma_{k}\right)=\mathbb{Z}\left[x_{k}, y_{k}, z_{k}\right], \operatorname{dim} x_{k}=$ $\operatorname{dim} y_{k}=2, \operatorname{dim} z_{k}=4$ subject to the relations: $2 x_{k}=2 y_{k}=x_{k} y_{k}=$ $2^{k} z_{k}=0, x_{k}^{2}=y_{k}^{2}=2^{k-1} z_{k}$. 


\section{REFERENCES}

[1] J. F. Adams, Stable Homotopy and Generalized Homology, University of Chicago Mathematics Lecture Notes, 1971.

[2] M. F. Atiyah, Characters and cohomology of finite groups, I.H.E.S. Publ. Math., 9 (1961), 23-64.

[3] N. A. Baas, On the Stable Adams Spectral Sequence, Aarhus Universitët Lecture Notes, 1969.

[4] T. Bröcker and T. t. Dieck, Kobordismen Theorie, Lecture Notes in Math., Vol. 178, Springer Verlag, 1970.

[5] H. Cartan and S. Eilenberg, Homological Algebra, Princeton University Press, 1956.

[6] C. W. Curtis and I. Reiner, Representation Theory of Finite Groups and Associative Algebras, Wiley, New York, 1962.

[7] T. t. Dieck, Steenrod operationen in kobordismen theorien, Math. Z., 107 (1968), 380-401.

[8] $\longrightarrow$ Bordism of G-manifolds and integrality theorems, Topology, 9 (1970), 345-358.

[9] _ Actions of finite Abelian p-groups without stationary points, Topology, 9 (1970), 359-366.

[10] D. Pitt, Free actions of generalized quaternion groups on spheres, Proc. London Math. Soc., 26 (1973), 1-18.

[11] E. H. Spanier, Algebraic Topology, McGraw-Hill, 1966.

[12] R. E. Stong, Notes on Cobordism Theory, Mathematical Notes, Princeton University Press, 1968.

[13] P. S. Landweber, Coherence, flatness and cobordism of classifying spaces, Proc. Adv. Study. Inst. Alg. Top. 256-269, Aarhus 1970.

[14] D. C. Ravenel, Complex Cobordism and Stable Homoty Groups of Spheres, Academic Press, Inc., 1986.

Received October 5, 1986 and in revised form August 15, 1988.

\section{UNIVERSITÉ MOHAMMED V}

B.P. 1014

RABAT, MOROCCO 



\section{PACIFIC JOURNAL OF MATHEMATICS EDITORS}

\author{
V. S. VARADARAJAN \\ (Managing Editor) \\ University of California \\ Los Angeles, CA 90024-1555-05 \\ Herbert Clemens \\ University of Utah \\ Salt Lake City, UT 84112 \\ ThOMAS ENRIGHT \\ University of California, San Diego \\ La Jolla, CA 92093
}

\section{R. FINN}

Stanford University

Stanford, CA 94305

HeRmann FlaschKa

University of Arizona

Tucson, AZ 85721

VAUGHAN F. R. JONES

University of California

Berkeley, CA 94720

Steven KerckhofF

Stanford University

Stanford, CA 94305

\author{
RobION KIRBY \\ University of California \\ Berkeley, CA 94720 \\ C. C. Moore \\ University of California \\ Berkeley, CA 94720 \\ HAROLD STARK \\ University of California, San Diego \\ La Jolla, CA 92093
}

\section{ASSOCIATE EDITORS}
R. ARENS
E. F. BECKenBach
B. H. NeumanN
F. WOLF
K. YoshidA (1906-1982)
(1904-1989)

\section{SUPPORTING INSTITUTIONS}

UNIVERSITY OF ARIZONA

UNIVERSITY OF BRITISH COLUMBIA

CALIFORNIA INSTITUTE OF TECHNOLOGY

UNIVERSITY OF CALIFORNIA

MONTANA STATE UNIVERSITY

UNIVERSITY OF NEVADA, RENO

NEW MEXICO STATE UNIVERSITY

OREGON STATE UNIVERSITY
UNIVERSITY OF OREGON

UNIVERSITY OF SOUTHERN CALIFORNIA

STANFORD UNIVERSITY

UNIVERSITY OF HAWAII

UNIVERSITY OF TOKYO

UNIVERSITY OF UTAH

WASHINGTON STATE UNIVERSITY

UNIVERSITY OF WASHINGTON 


\section{Pacific Journal of Mathematics}

Vol. 142, No. $1 \quad$ January, 1990

Marco Andreatta, Mauro Beltrametti and Andrew Sommese, Generic properties of the adjuction mapping for singular surfaces and applications

Chen-Lian Chuang and Pjek-Hwee Lee, On regular subdirect products of

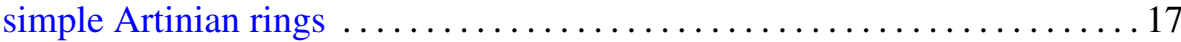

Fernando Giménez and Vicente Miquel Molina, Volume estimates for real hypersurfaces of a Kaehler manifold with strictly positive holomorphic sectional and antiholomorphic Ricci curvatures $\ldots \ldots \ldots \ldots \ldots \ldots 23$

Richard J. Griego and Andrzej Korzeniowski, Asymptotics for certain Wiener integrals associated with higher order differential operators

Abdeslam Mesnaoui, Unitary bordism of classifying spaces of quaternion

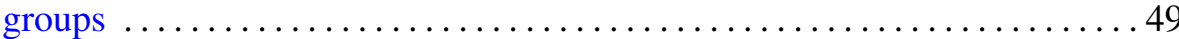

Abdeslam Mesnaoui, Unitary cobordism of classifying spaces of quaternion

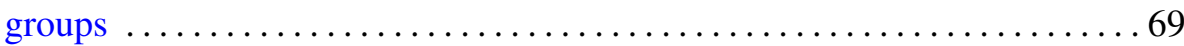

Jesper M. Møller, On equivariant function spaces $\ldots \ldots \ldots \ldots \ldots \ldots \ldots \ldots$

Bassam Nassrallah, A $q$-analogue of Appell's $F_{1}$ function, its integral representation and transformations

Peter A Ohring, Solvability of invariant differential operators on metabelian

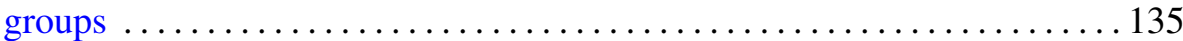

Athanase Papadopoulos and R. C. Penner, Enumerating pseudo-Anosov foliations

Ti-Jun Xiao and Liang Jin, On complete second order linear differential

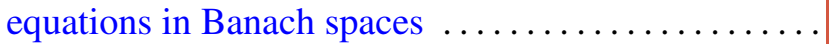

Carl Widland and Robert F. Lax, Weierstrass points on Gorenstein curves 